\title{
Affine differential geometry analysis of human arm movements
}

\author{
Tamar Flash • Amir A. Handzel
}

Received: 23 May 2006 / Accepted: 20 February 2007 / Published online: 4 April 2007

(C) Springer-Verlag 2007

\begin{abstract}
Humans interact with their environment through sensory information and motor actions. These interactions may be understood via the underlying geometry of both perception and action. While the motor space is typically considered by default to be Euclidean, persistent behavioral observations point to a different underlying geometric structure. These observed regularities include the "two-thirds power law", which connects path curvature with velocity, and "local isochrony", which prescribes the relation between movement time and its extent. Starting with these empirical observations, we have developed a mathematical framework based on differential geometry, Lie group theory and Cartan's moving frame method for the analysis of human hand trajectories. We also use this method to identify possible motion primitives, i.e., elementary building blocks from which more complicated movements are constructed. We show that a natural geometric description of continuous repetitive hand trajectories is not Euclidean but equi-affine. Specifically, equi-affine velocity is piecewise constant along movement segments, and movement execution time for a given segment is proportional to its equi-affine arc-length. Using this mathematical framework, we then analyze experimentally recorded drawing movements. To examine movement segmentation and classification, the two fundamental equi-affine differential invariants-equi-affine arc-length and curvature are calculated for the recorded movements. We also discuss the possible role of conic sections, i.e., curves with constant equi-affine curvature, as motor primitives and focus in more detail on parabolas, the
\end{abstract}

T. Flash $(\bowtie) \cdot$ A. A. Handzel

Department of Computer Science and Applied Mathematics,

Weizmann Institute of Science, Rehovot, 76100 Israel

e-mail: tamar.flash@weizmann.ac.il

A. A. Handzel

e-mail: ahandzel@ieee.org equi-affine geodesics. Finally, we explore possible schemes for the internal neural coding of motor commands by showing that the equi-affine framework is compatible with the common model of population coding of the hand velocity vector when combined with a simple assumption on its dynamics. We then discuss several alternative explanations for the role that the equi-affine metric may play in internal representations of motion perception and production.

\section{Introduction}

How do humans and animals recognize an object when it undergoes a certain transformation such as rotation or translation? How do they recognize and imitate movements or gestures made by others when the observer's viewpoint changes? In vision research, these and similar questions have led to a major interest in geometrical aspects of visual perception and object recognition based on the identification of different types of invariants (e.g., algebraic, differential). While similar also arise in the study of motor control, less emphasis has been placed on a rigorous investigation of geometrical aspects of motion planning and control. Thus, in recent years greater efforts have been directed at evaluating similarities and differences among different movement instantiations, when performed by the same individual within different contexts or by different individuals. Nevertheless, a greater focus on the identification of motor invariants may help in gaining further insight into how movements are internally represented and stored in memory and how these internal representations are used to successfully generate the entire motor repertoire.

A particularly important question in this regard is what spatial and temporal variables are used by such internal representations (Stein et al. 1985; Wolpert and Ghahramani 
2000; Flash and Sejnowski 2001). Inevitably, these variables must be associated with both kinematic and dynamic aspects of movement generation. Nevertheless, many recent load adaptation studies have demonstrated that sufficient practice in the presence of external dynamic loads restores the original kinematic and spatial aspects of the movements (Flash and Gurevich 1997; Shadmehr and Mussa-Ivaldi 1994), even though this may require significant modifications of muscle activation patterns (Padoa-Schioppa et al. 2004). These observations suggest that within the nervous system there exist central representations which are primarily concerned with more abstract geometric and temporal attributes of the movements, rather than with motor execution or muscle activations.

Capacity constraints make it highly unlikely that the commands to all possible movement instantiations are stored in memory in the form of a motor tape. The motor system seems to rely on much more parsimonious representations of motor information. One line of evidence favoring such parsimony of representation is the existence of certain spatial and temporal features of the movement that are kept invariant under different spatial and temporal transformations, such as translation, rotation, amplitude and speed scaling. Support for the existence of motor invariants is derived from numerous studies of both reaching and curved movements (Lacquaniti 1989; Flash and Hogan 1987; Viviani and Flash 1995; Richardson and Flash 2002), and more complicated sequential behaviors, e.g., scribbling (Soechting and Terzuolo 1987a,b), writing (Lacquaniti 1989), drawing (Viviani and Cenzato 1985; Viviani and Flash 1995) and typing (Soechting and Flanders 1997). These observations suggest that the motor system may store a much more limited set of templates or motor prototypes, as in a motor alphabet, and that the rich repertoire of human and animal movements is generated by applying certain basic operations or transformations to this basic set of primitives. These, in turn, are joined together using some form of syntactic rules. For reviews see Mussa-Ivaldi and Solla (2004), Poggio and Bizzi (2004), Flash and Hochner (2005).

A series of neurophysiological and behavioral studies support the existence of motor primitives at the more peripheral levels which are involved in motor execution (MussaIvaldi and Solla 2004). The evidence for the existence of motor primitives at the kinematic or more abstract levels is less direct and derives from developmental studies in infants (Von-Hofsten 1991; Berthier 1996), human arm trajectory modification and target interception studies (Flash and Henis 1991; Milner 1992), patient studies (Krebs et al. 1999; Rohrer et al. 2002), and studies dealing with motor learning through practice (Sosnik et al. 2004) or through imitation (Schaal et al. 2003). In spite of earlier efforts, mostly directed at empirically inferring the underlying motor primitives, we still lack conceptual and theoretical formalisms or approaches for examining the validity of the existence of motor primitives and whether the entire repertoire of possible actions can be derived from a limited set of such elementary units. Investigating these ideas raises several questions of fundamental significance:

1. Is it possible to identify a certain motor alphabet from which more elaborate "syllables", words and sentences can be composed?

2. What are the characteristics of such motor elementary units and how are they internally coded? What makes each primitive distinctly different from the others?

3. What attributes or invariants can assist us in establishing whether two apparently different movements were generated from the same basic element by applying differently parameterized but qualitatively similar transformations?

4. What movement generation rules (basic operations or transformations) are used to generate a large repertoire of movements from relatively few elements?

5. What syntactic rules are used by the motor system to join motor elements or units of action?

Here, focusing mainly on the first three questions, we illustrate how the language of differential geometry and Lie algebra is particularly suitable for addressing these issues.

In particular, studies of drawing and scribbling movements have led to the discovery of several particular "conservation laws" in human movement. One of these laws is the twothirds power law, formulated by Lacquaniti et al. (1983) to account for the puzzling coupling between Euclidean velocity and curvature empirically observed during 2D handwriting and drawing movements (Viviani and Cenzato 1985; Flash and Hogan 1987; Edelman and Flash 1987; Soechting and Terzuolo 1987b; Viviani and Flash 1995; Sternad and Schaal 1999). In earlier studies Pollick and Sapiro (1997) and Flash and Handzel (1996) have independently shown that the two-thirds power law is compatible with movements being generated with a piecewise constant equi-affine velocity. The purpose of the present study is to use this discovery as a starting point for further investigations aimed at characterizing motor primitives and their internal representations.

In particular, we first discuss the importance of identifying what possible metrics are used for internally representing arm movements and show how the equi-affine distance is a more appropriate metric than the Euclidean one. Under affine transformations different segments of a curve can be stretched to different degrees along the horizontal and vertical directions but parallel lines remain parallel. The equi-affine transformation is a more restricted transformation that also preserves the area of the parallelogram defined by the equiaffine tangent and equi-affine normal to the curve. We show that the two-thirds power law is compatible with an internal representation in which the equi-affine velocity of the movements being planned and generated is piecewise constant. 
We make then further use of the equi-affine geometrical framework to develop a mathematical formalism that will allow us to better analyze and characterize human arm movements. In particular, we derive equi-affine differential invariants and signature curves of the observed movements which can be used for the identification and classification of motion primitives and for movement segmentation. We also show that the equi-affine velocity being piecewise constant may reflect the characteristics of the neural code used by cortical cells to represent the movement. Finally we discuss the relationship between the use of equi-affine arc-length as the internal metric for motor representation, the isochrony principle and the time scaling property of human movements.

\subsection{Basic characteristics of human drawing and scribbling} movements

It has often been suggested that in motor tasks, such as handwriting or drawing, the motor system need not internally represent all possible letters or figural forms but may instead use a limited set of basic primitives or strokes that are then concatenated to form more complicated movements (Lacquaniti 1989). Such motion segments have been identified by detecting changes in movement curvature, tangential velocity or the coefficient relating these two variables to each other, as in the two-thirds power law (Viviani and Cenzato 1985; Viviani 1986). Thus, in spite of the presumable independence between the geometrical form of the trajectory and the velocity of movement along the path, evidence has accumulated pointing to the inherent dependence between these two variables during human and monkey movements. In particular, in naturally executed curved and drawing movements, the angular velocity decreases with increasing curvature and is proportional to the two-thirds power of the latter (Lacquaniti et al. 1983) as follows:

$A=G \times k^{2 / 3}$

where $A$ is the angular velocity and $k$ is the movement curvature. An equivalent formulation of this expression, called the one third power law, is: $v(t)=G r^{1 / 3}$ or $k \times v^{3}=G$ where $r=1 / k$ is the Euclidean radius of curvature and $v$ is the Euclidean velocity, i.e., the derivative of the Euclidean arc-length $s$ with respect to time $t$, i.e., $v=\frac{\mathrm{d} s}{\mathrm{~d} t}=\frac{A}{k}$. The gain factor of the above relationship, $G$, which was called the velocity gain factor, was found to be piecewise constant and to be determined by the linear extent of each segment. It was also suggested that hand velocity during any particular movement need not be explicitly coded but may be automatically derived from the coupling between speed and curvature, as expressed by the two-thirds power law (Lacquaniti et al. 1983).

Different explanations, either at the kinematic, dynamic or neural levels have been suggested for this law. Optimization principles, such as smoothness maximization (Flash and Hogan 1987; Richardson and Flash 2002) or variance minimization (Harris and Wolpert 1998), can produce the observed relationship between geometrical form and velocity. Others have suggested that this power law may reflect the mechanical properties of the neuromuscular system, such as the low-pass filtering properties of muscles (Gribble and Ostry 1996). Nevertheless, recent neurophysiological findings (Schwartz 1992, 1993, 1994; Moran and Schwartz 1999a,b; Schwartz and Moran 1999) have demonstrated that the law does reflect central control mechanisms and not merely low-level mechanical factors. Finally, de'Sperati and Viviani (1997) have shown that smooth pursuit eye movements also obey the two-thirds power law.

The temporal aspect of movement generation has not been sufficiently explored beyond the context of Fitt's law. Nevertheless, three important features of human arm trajectories described in earlier studies are global and local isochrony (Viviani 1986; Viviani and Cenzato 1985; Viviani and Flash 1995) and the scaling property of trajectories with respect to speed. Global isochrony is associated with the relative insensitivity of the total movement duration to changes in the overall size of the trajectory. Another feature of human movements is local isochrony, namely the modulation of speed within individual movement segments according to their Euclidean amplitude, thus keeping the duration of each individual segment relatively insensitive to its length. Finally, a third feature of human movements relates to their scaling property with respect to speed (Hollerbach and Flash 1982; Atkeson and Hollerbach 1985), namely that the instantaneous speed is scaled by a multiplicative factor, whose value depends on the ratio of the overall movement duration to that of some reference movement.

\subsection{Affine metrics, differential invariants and motor primitives}

Beyond our wish to account for the $2 / 3$ power law, our more general objective is to develop a mathematical framework, rooted in geometry, which will allow us to examine the nature of motion primitives. Such a framework may enable us to investigate whether different movements that belong to the same subclass, are generated from the same template by a parametrically different but qualitatively similar set of operations. We found the language of differential geometry and Lie algebra (Olver 1993) to be especially suitable for developing such a mathematical framework. With this language we can now seek a group of actions or operations where we can unravel one particular trajectory by repeatedly applying a single member from this group of motions to some geometrical element, representing, say, instantaneous hand position and orientation. Similarly, it is possible to generate an entire family of trajectories from one orbit or template by each 
time applying a different member from that particular group of motions or transformations. More specifically, a whole movement along either a 2D or 3D curve might be generated by varying the value of the arc-length parameter, be it Euclidean or equi-affine. The arc-length parameter therefore constitutes the group's metric. Similarly, it is possible to generate a whole family of orbits or trajectories by each time applying a different element or member of the Euclidean or equi-affine group of motions, while keeping some attributes or characteristics of the movement invariant modulo the group transformation. Below we discuss two terms which are of crucial importance for the discussion of internal representations of motion-metrics and differential invariants.

\subsubsection{Internal metrics}

The notion of a metric with respect to which movements are planned, internally coded, or selected is of crucial importance for motor control. A metric is related to the ability to define "a distance" between two different states within a metric space. A metric space is a set $\mathbf{S}$ equipped with a distance, that is a function $d$ mapping of $\mathbf{S} \times \mathbf{S}$ into $\mathbf{R}$ and satisfying for all $a, b, c, d \in \mathbf{S}$ :
1. $\quad d(a, b) \geq 0$
2. $d(a, b)=0$ iff $a=b$
3. $d(a, b)=d(b, a)$
4. $\quad d(a, c) \leq d(a, b)+d(b, c)$

For example, Riemannian geometry is associated with the Riemannian metric, the norm induced by an inner product and is defined by $d s^{2}=g_{\alpha, \beta} d y^{\alpha} d y^{\beta}$. Although the notion of a metric is particularly important when discussing internal representations of either sensory or motor variables, very little is known about the metrics used by the motor or sensory systems. The few studies that have investigated the nature of such metrics in the context of motor control have tested whether there is an internally consistent spatial representation with, for example, a Riemannian structure (Fasse 1992).

The notion of a metric is also quite significant when assuming an underlying objective of the optimization of costs associated with performance of a motor task. We present evidence here that the metric used by internal motor representations is the equi-affine rather than the Euclidean metric. This is particularly interesting given the claim in vision research that visual perception is represented in terms of affine metrics. In a recent study, Todd et al. (2001) have shown that observers' judgements are systematically distorted relative to the physical environment but have instead an internally consistent affine structure. Here we will suggest that the equi-affine metric might be more appropriate than the Euclidean metric for describing the neural code subserving the representations of arm (and perhaps also eye) trajectories.

\subsubsection{Differential invariants}

Invariants, particularly differential invariants, are a highly pertinent concept for deciding whether a series of movements was generated from the same prototype or primitive by using each time a different action or transformation from the same group of motions. In vision research, certain symmetry groups and their associated differential invariants have assumed a great significance in the study of shape recognition and image processing. One such problem is the recognition of a curve or planar object that may be partially occluded and has been transformed by a geometric viewing condition. This common visual recognition task is naturally based on the use of different invariants under various groups of viewing transformations. Given the difficulties associated with the use of algebraic invariants, the use of differential invariants, which are local and are determined for each point on a curve, was suggested as an alternative. Hence, just as the ordinary invariants of a group action serve to characterize invariant equations, differential invariants completely characterize invariant systems of differential equations for the group, as well as invariant variational principles. Differential invariants, therefore, form the basis for many physical theories where one begins by postulating an invariance of differential equations or a variational principle under the action of a particular symmetry group. In particular, in recent years differential invariants were found to be highly significant in the study of curve evolution based on the use of invariant heat-flow type diffusion equations (Sapiro and Tannenbaum 1994).

Here, we are interested in how a geometry induced by a transformation group is applied to a smooth curve or a trajectory. In describing a given trajectory, it is important to distinguish between the geometric concept of a plane curve and its parametric description. It is, therefore, useful to consider the image (or trace) of the curve $C(p)$ denoted by $\operatorname{Img}[C(p)]$ (or the equivalent path, using motor control terminology). Hence, if a curve $C(p)$ is parameterized by a new parameter $w$ such that $w=w(p), \frac{\partial p}{\partial w} \geq 0$, we obtain that $\operatorname{Img}[C(p)]=\operatorname{Img}[C(w)]$. In general, the parametrization through a parameter $\mathrm{p}$ gives the "velocity" of the trajectory. Given a transformation group $R$, the curve can be parameterized by what is called the arc-length, $d r$ which is an invariant of the group and is useful for defining differential invariant descriptors (Olver et al. 1994). To perform this parametrization the group metric, $g$, is defined by the expression: $\mathrm{d} r=g \mathrm{~d} p$ for any parametrization $p$ where $r$ is obtained via the relation: $r=\int_{0}^{p} g(\xi) \mathrm{d} \xi$. We have, of course, $\operatorname{Img}[C(p)]=\operatorname{Img}[C(r)]$. Based on the group metric and arc-length, the group curvature can be computed using either Lie theory or Cartan's moving frame method (Cartan 1935; Faugeras 1993). The group curvature as a function of arc-length is defined as the simplest non-trivial 
differential invariant of the group. The fact that for transitive group actions, an object can be fully reconstructed modulo group transformations from a prescribed and finite collection of differential invariants is a consequence of a general theorem by Elie Cartan (Cartan 1935; Faugeras 1993; Calabi et al. 1996).

In the Euclidean case, for example, we have $g_{\text {euc }}=\left\|\frac{\partial \mathbf{C}}{\partial \mathbf{p}}\right\|$ and the Euclidean arc-length is given by $s=\int_{0}^{p}\left\|\frac{\partial \mathbf{C}}{\partial \xi}\right\| \mathrm{d} \xi$. This parametrization is an Euclidean invariant since the norm is invariant. The simplest differential invariant in the Euclidean case, is the Euclidean curvature which is invariant under the Euclidean group of motions consisting of translations and rotations. A curve in Euclidean space is uniquely determined modulo translation and rotation by its invariant curvature and the first derivative of curvature with respect to Euclidean arclength. Hence, it is fully prescribed by its Euclidean signature curve, which is parameterized by the Euclidean curvature and its derivative with respect to arc-length.

Here we consider another group of motions, namely equiaffine transformations. The affine arc-length $\sigma$ is defined as $\sigma=\int k^{\frac{1}{3}} \mathrm{~d} s$ where $s$ and $k$ are the Euclidean arc-length and curvature, respectively. A curve is the affine plane is fully prescribed by its affine signature curve which is parameterized by the affine curvature and its derivative with respect to the affine arc-length (Calabi et al. 1996; Calabi et al. 1998).

Below we describe the methods used to derive both the equi-affine arc-length and equi-affine velocity and the signature curves for several characteristic hand trajectories. We also give a more formal description of Cartan's moving frame method for deriving differential invariants and then show that the two-thirds power law is consistent with the equi-affine velocity being piecewise constant. We then define the equiaffine curvature and describe the trajectories corresponding to constant equi-affine curvature. In particular, we describe the mathematical expressions for parabolas, hyperbolas and ellipses, which have constant equi-affine curvature. We note that parabolas are affine geodesics that maximize the affine arc-length. Given the interesting relationship between affine velocity, the velocity gain factor and proper time, we show for movements that obey the two-thirds power law, how speed scaling and the isochrony principles can be expressed using the affine description. Finally we describe the numerical procedures used to analyze experimentally measured arm movements, using the affine differential geometry tools developed here.

\section{Affine geometry of arm movements}

Here we introduce a new approach to the study of the arm movements of humans and other primates. The novelty of our method lies in viewing the internal representation of the extrinsic space associated with arm motion generation as having a geometric structure different from the usual Euclidean geometry. Before elaborating our approach, we set out the scope and context of this study.

In usual motor behavior-whether point-to-point movements such as reaching or continuous motion such as drawing - the focus is on the position of the hand, which is referred to as the end effector. In certain circumstances the end effector may be the elbow or the point of a tool held in the hand (Tanaka et al. 1996). However, we shall not deal with these cases. Since the time-dependent position of the hand is of prime importance, it is common to study the trajectory that the hand traces in space while ignoring the limb and the body. Despite this simplification, mathematical analysis of endpoint trajectories does provide information about the motor system.

The trajectories of the hand in natural reaching and continuous drawing-like movements are very often restricted to one plane or are often approximately piecewise planar in space (Soechting and Terzuolo 1987a). This allows us to reduce the analysis of a large class of movements to arbitrarily oriented planes in space. For reasons explained below, we replace the traditional Euclidean view of these planes by viewing them as affine planes, $\mathbf{A}^{2}$. The properties of affine geometry are quite different from those of Euclidean geometry, as we review in the Sect. 3, and give rise to new results in the analysis of human movement. Our work here grew from the initial observation that the empirical rule known as the "one-third power law" relating hand velocity to path curvature is equivalent to movement with piecewise constant equi-affine speed (Flash and Handzel 1996; Pollick and Sapiro 1997). An important implication of this finding is that in a large class of hand movements the natural setting is actually affine geometry, and this provided the initial motivation for the current work.

This equivalence is demonstrated in Sect. 3.5. This is followed by Sect. 4 in which we discuss local isochrony and the speed scaling property of human movements. In Sect. 5 we focus on the analysis and mathematical description of parabolic segments, the equi-affine geodesics. Finally, in Sect. 6 we describe the application of recently developed affine invariant numerical tools to the analysis of hand trajectories when tracing stereotyped figural forms.

\section{Background: affine plane geometry}

We now review the theory of curves in the affine plane, starting with a brief description of the plane itself, emphasizing how it differs from the Euclidean plane (Olver et al. 1996). We then present the method of the moving of a curve, an ingenious formalism developed by Élie Cartan (Cartan 1935; Faugeras 1993; Guggenheimer 1977; Spivak 1979). Its main theme is the relation between a curve and the action of a Lie 
group of transformations. Given such a group, we look for a parametrization of curves which is invariant under the group action. This leads to two significant results. Locally, we can think of the curve as "unraveling" under the local action of the group. Globally, each curve is a member of an infinite family of curves, any pair of which is related by a transformation of the group. Each family of curves can be represented in a unique way by invariant functions-either the associated curvature function, or a signature curve. Applications of these representations are discussed below.

\subsection{The affine plane}

Affine geometry is, loosely speaking, Euclidean geometry stripped of its metric structure, i.e., without means of measuring distances or angles. A more rigorous explanation of non-metric or pure affine space is given below. The space can be endowed with a metric structure; if the latter conforms to the regular Euclidean distance, it gives rise to Euclidean geometry. In what follows, we refer to $p$ lane affine geometry.

Affine geometry contains two distinct spaces (for further details see Calabi et al. 1996). The basic plane $\mathbf{A}^{2}$ comprises the same points as $\mathbf{R}^{2}$, but it does not have the structure of a vector space: the affine plane has no preferred point designated as the origin, two points cannot be added, and a scalar product is not defined on it. The second space is a plane of displacements, $V$, which is a vector space. The basic operation in the affine plane is subtraction, where the difference between two points $p_{1}, p_{2} \in \mathbf{A}^{2}$ is a vector in the displacement plane:

$p_{2}-p_{1}=\mathbf{v} \in V$,

which is sometimes denoted as $p_{1} p_{2}$. Such a vector, in turn, maps points in the affine plane to other points by translation:

$p_{1}+\mathbf{v}=p_{2}$.

An affine coordinate system on $\mathbf{A}^{n}$ is determined by $n+1$ affinely independent points $\left(p_{0}, p_{1}, \ldots, p_{n}\right)$ meaning that the $n$ displacement vectors $\mathbf{a}_{i}=p_{i}-p_{0}$ constitute a basis for $V$.

The vector space $V$ is canonical in the sense that the tangent plane $T_{p} \mathbf{A}^{2}$ at each point can be identified with it. For any $n$-dimensional vector space one can take antisymmetric products of $k$ vectors, called a wedge product which is a generalization of the binary cross product to the multi-variable case (Spivak 1979). The space of all possible combinations of $k$-products, for $k$ running from 0 to $n$, is the exterior algebra over $V$ (Conlon 1993). Its subspace of $k$-products for a particular $k$ is denoted by $\bigwedge^{k} V$. For $k=$ $n, \bigwedge^{n} V$ is a one dimensional space of determinant forms, which serves to define volume over $V$. Each basis $\left\{\mathbf{e}_{i}\right\}$ of $V$ determines an $n$-dimensional parallelotope whose volume is $\mathbf{e}_{1} \wedge \mathbf{e}_{2} \wedge \cdots \wedge \mathbf{e}_{n} \in \wedge^{n} V$; a unit volume can thus be fixed by choosing such a basis. Denoting by square brackets the volume determined by $n$ independent vectors relative to a chosen basis: $\mathbf{a}_{i}=\sum x_{i}^{j} \mathbf{e}_{j}$, the volume is given by the determinant of the matrix of the vector components (the wedge product of $n$ of $n$ vectors). For the affine plane, in particular, a pair of non-collinear vectors determines the area of a parallelogram

$\left[\mathbf{a}_{1}, \mathbf{a}_{2}\right]=\operatorname{Det}\left(\begin{array}{ll}a_{1}^{1} & a_{2}^{1} \\ a_{1}^{2} & a_{2}^{2}\end{array}\right)$

where superscripts denote the components of a vector.

As mentioned above, the pure affine structure does not possess a measure of distances or lengths; instead the volume in $3 \mathrm{D}$ or the area in 2D serve as fundamental quantities. Nevertheless, a rudimentary form of length measurement exists as the ratio between segments of parallel lines. This property will be used below.

Strictly speaking, affine plane geometry comprises the pair $\left(\mathbf{A}^{2}, V\right)$, but the short notation $\mathbf{A}^{2}$ will be used here, keeping in mind that it represents the complete structure. We conclude with a useful notion: An affine element is a vector which is anchored at a point, namely a pair $(p, \mathbf{v})$. Affine elements can be viewed as the constituent objects of affine spaces similarly to points in Euclidean spaces.

\subsection{The Euclidean moving frame of a curve}

After recalling the pertinent notions of affine spaces we are ready to discuss curves in the affine plane, but since this requires the introduction of additional non-trivial structure, it is instructive to look first at curves in the more familiar Euclidean plane.

A curve $\gamma(t)$ in the plane is parameterized by a mapping

$$
\begin{aligned}
& \gamma: I \longrightarrow \mathbf{R}^{2} \\
& \gamma: t \longmapsto \mathbf{x}(t)=\left\{x_{1}(t), x_{2}(t)\right\},
\end{aligned}
$$

where $t \in I=[0,1]$ and $x_{i}$ is the $i$-th Cartesian coordinate. The parametrization by time $t$ is not unique-the same set of points in the plane that constitutes the curve can be produced by an infinity of different maps $\gamma(t)$ where $t$ can be replaced by any diffeomorphism $h(t)$ of $I$ (e.g., $t \mapsto t^{2}$ ). The tangential velocity (i.e., tangent vector) to the curve is the velocity

$\mathbf{v} \equiv \dot{\mathbf{x}}(t)=\left(\dot{x}_{1}(t), \dot{x}_{2}(t)\right)$,

where the dot denotes differentiation w.r.t. the parameter $t$; the speed is

$\|\mathbf{v}\|=\left(\dot{x}_{1}^{2}(t)+\dot{x}_{2}^{2}(t)\right)^{\frac{1}{2}}$. 


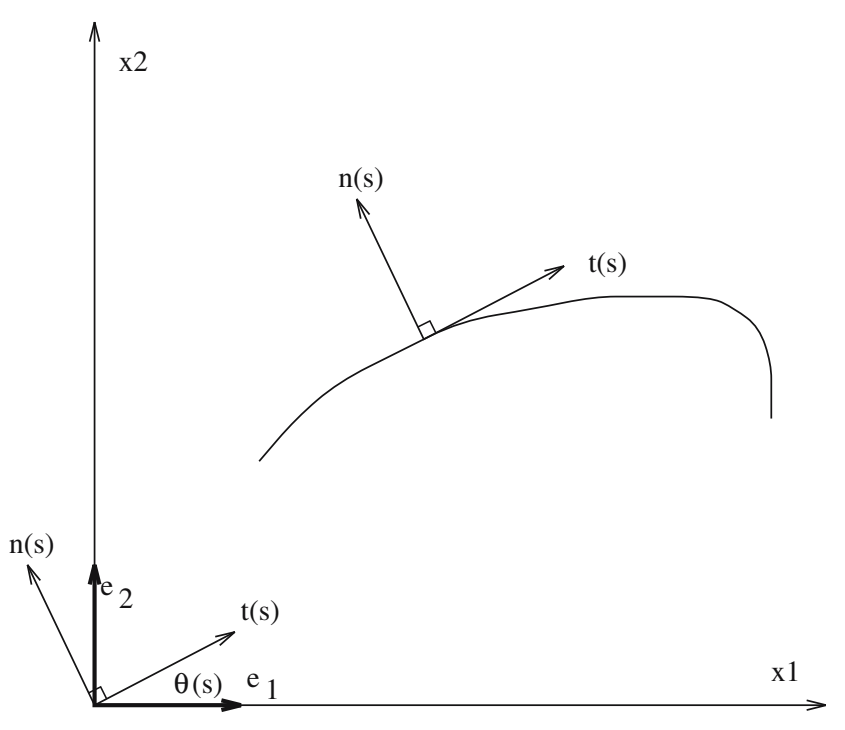

Fig. 1 The Euclidean moving frame: the velocity and acceleration constitute the directions of a Cartesian coordinate system $\{\mathbf{t}, \mathbf{n}\}$ (the tangent and the normal to it) at each point $\mathbf{x}(s)$ on a curve which is parameterized by arc-length $s$. The frame can be thought to be rotating along the curve relative to a fixed system $\left\{\mathbf{e}_{1}, \mathbf{e}_{2}\right\}$ as the curve evolves. The angle between $\mathbf{t}$ and $\mathbf{e}_{1}$ is $\theta(s)$

There is, however, a special parametrization $s$ of the curve, called the arc-length, which can be defined implicitly as the length for which the speed equals 1 along the whole curve:

$\left\|\mathbf{x}^{\prime}(s)\right\|=\|\mathbf{v}(s)\| \equiv 1$

where differentiation w.r.t. arc-length is denoted by prime in order to distinguish it from the differentiation w.r.t. any other parametrization of the curve. The change from $t$ to $s$ implies the replacement of $I$ by some other interval $[0, L]$. With the arc-length parametrization, velocity changes only in direction and not in length and so the acceleration $\mathbf{x}^{\prime \prime}(s)$ is always perpendicular to velocity. To each point $\mathbf{x}(s)$ on the curve we can therefore attach a local orthonormal coordinate system $\{\mathbf{t}, \mathbf{n}\}$ called a moving frame, with $\mathbf{t}$ being the direction of tangential velocity and $\mathbf{n}$ the direction of acceleration, as depicted in Fig. 1 after Guggenheimer (1977). The tangent $\mathbf{t}$ is rotated by angle $\theta$ relative to a fixed external frame $\left\{\mathbf{e}_{1}, \mathbf{e}_{2}\right\}$; as the curve evolves with $s$, this angle changes too, i.e., $\theta=\theta(s)$.

Using a rotation matrix $R(\theta)$ for the moving frame in the fixed frame coordinates, we have

$\{\mathbf{t}, \mathbf{n}\}=R(\theta)\left\{\mathbf{e}_{1}, \mathbf{e}_{2}\right\}$.

An infinitesimal rotation of the frame along the curve is determined by

$$
\frac{\mathrm{d}}{\mathrm{d} s}\{\mathbf{t}, \mathbf{n}\}=R^{\prime}\left\{\mathbf{e}_{1}, \mathbf{e}_{2}\right\}=R^{\prime} R^{-1}\{\mathbf{t}, \mathbf{n}\}
$$

This gives the matrix form of the Frenet equations of the Euclidean plane:

$\{\mathbf{t}, \mathbf{n}\}^{\prime}=\mathcal{C}(R)\{\mathbf{t}, \mathbf{n}\}$,

where $\mathcal{C}(R) \equiv R^{\prime} R^{-1}$ is the Cartan matrix of the rotation $R$, thereby eliminating the external fixed frame and emphasizing the local intrinsic character of this motion. In explicit form, the Cartan matrix is

$\mathcal{C}(R)=\left(\begin{array}{cc}0 & k(s) \\ -k(s) & 0\end{array}\right)=k(s) J$

where

$J=\left(\begin{array}{rr}0 & 1 \\ -1 & 0\end{array}\right)$

and $k(s)=\theta^{\prime}(s)$ is the curvature. The Cartan matrix is an element of the Lie algebra $\mathfrak{s o}(2)$, the generator of the group of rotations in the plane, $S O(2)$. Notice that this group comprises the set of possible transformations between frames.

The explicit form of the Euclidean curvature is

$k(s)=\left|\mathbf{x}^{\prime}(s) \wedge \mathbf{x}^{\prime \prime}(s)\right|=\left|\dot{x}_{1}(s) \ddot{x}_{2}(s)-\dot{x}_{2}(s) \ddot{x}_{1}(s)\right| ;$

and for a general parametrization $t$ :

$k(t)=\frac{\left|\dot{x}_{1}(t) \ddot{x}_{2}(t)-\dot{x}_{2}(t) \ddot{x}_{1}(t)\right|}{\|\dot{\mathbf{x}}(t)\|^{3}}$.

The Euclidean curvature as a function of arc-length provides an invariant representation of the curve in the Euclidean plane. This issue will be dealt with in more detail after we examine curves in the affine plane.

\subsection{The affine moving frame}

We now turn to curves in the affine plane, $\mathbf{A}^{2}$ and make our way from Euclidean to affine structure via the moving frame. We relax the restriction that a frame be composed of a pair of orthonormal vectors and require only that the vectors be linearly independent.

Definition 1 An affine frame is a pair of non-collinear vectors attached to a point $\mathbf{x} \in \mathbf{A}^{2}$, i.e.,

$\left(\mathbf{x} ; \mathbf{a}_{1}, \mathbf{a}_{2}\right), \quad \mathbf{a}_{1}, \mathbf{a}_{2} \in T_{x}\left(\mathbf{A}^{2}\right)$,

where $T_{x}\left(\mathbf{A}^{2}\right)$ is the tangent plane at the point $\mathbf{x}$.

The non-collinearity is required in order for a frame to exist and allows defining a volume - an area for the planeaccording to Eq. (2):

$\Omega\left(\mathbf{a}_{1}, \mathbf{a}_{2}\right)=\mathbf{a}_{1} \wedge \mathbf{a}_{2}=\left[\mathbf{a}_{1}, \mathbf{a}_{2}\right]=1$. 
Each pair of frames that obey Eq. (15) are related by an element of the unimodular affine group $G=S L(2)$, i.e., areapreserving linear transformations of the plane. This group is larger than the rotation group and, thus, by easing the restriction on what constitutes a frame, we also obtain more flexibility in the transformations acting on them.

Now we write the affine frame vectors in terms of the parameterized curve:

$\mathbf{a}_{1}=c \dot{\mathbf{x}}(t)$

$\mathbf{a}_{2}=c \ddot{\mathbf{x}}(t)$

where

$c=|\dot{\mathbf{x}}(t) \wedge \ddot{\mathbf{x}}(t)|^{-\frac{1}{2}}=\left|\dot{x}_{1}(t) \ddot{x}_{2}(t)-\dot{x}_{2}(t) \ddot{x}_{1}(t)\right|^{-\frac{1}{2}}$

is a normalization factor in order to satisfy Eq. (15). The two frame vectors are parallel to the tangential velocity to the curve and to its acceleration. The affine frame matrix is

$A=c\left(\begin{array}{ll}\dot{x}_{1}(t) & \dot{x}_{2}(t) \\ \ddot{x}_{1}(t) & \ddot{x}_{2}(t)\end{array}\right)$

and it relates the local frame to an external fixed orthonormal system:

$\left\{\mathbf{a}_{1}, \mathbf{a}_{2}\right\}=A\left\{\mathbf{e}_{1}, \mathbf{e}_{2}\right\}$

similarly to Eq. (7) in the Euclidean case. As expected, the affine frame matrix belongs to the corresponding group, $A \in$ $S L(2)$. Its Cartan matrix is

$\mathcal{C}(A)=A^{\prime} A^{-1}=\left(\begin{array}{cc}-\frac{1}{2} \frac{|\dot{\mathbf{x}} \wedge \dddot{\mathbf{x}}|}{|\dot{\mathbf{x}} \wedge \ddot{\mathbf{x}}|} & 1 \\ -\frac{|\ddot{\mathbf{x}} \wedge \dddot{\mathbf{x}}|}{\mid \dot{\mathbf{x}} \wedge} & \frac{1}{2} \frac{\mid \dot{\mathbf{x}} \wedge}{|\dot{\mathbf{x}} \wedge \ddot{\mathbf{x}}|}\end{array}\right)$

and because Trace $\mathcal{C}(A)=0$, it lies in $\mathfrak{s l}(2)$ - the Lie algebra that generates the group $S L(2)$ - in analogy to the Euclidean case.

We wish to find an invariant parameterization of the matrix (and hence of the curve's Frenet equations) and, in the process, to equalize to zero as many coefficients of $\mathcal{C}(A)$ as possible. The diagonal elements can be set to zero if

$|\dot{\mathbf{x}} \wedge \dddot{\mathbf{x}}|=\frac{\mathrm{d}}{\mathrm{d} t}|\dot{\mathbf{x}} \wedge \ddot{\mathbf{x}}|=0$

In order to satisfy Eq. (20), we choose the parameter $\sigma$ for which

$\left|\mathbf{x}^{\prime} \wedge \mathbf{x}^{\prime \prime}\right| \equiv 1$

with the prime denoting derivation w.r.t. $\sigma$, which is called the affine arc-length. It is related to a general parameter $t$ through $|\dot{\mathbf{x}} \wedge \ddot{\mathbf{x}}|=\left|\mathbf{x}^{\prime} \wedge \mathbf{x}^{\prime \prime}\right| \dot{\sigma}^{3}=\dot{\sigma}^{3}$

where $\dot{\sigma}$ is the equi-affine speed of the curve. Thus the explicit expression for the affine arc-length is

$\sigma=\int_{\gamma} \mathrm{d} \sigma=\int|\dot{\mathbf{x}} \wedge \ddot{\mathbf{x}}|^{\frac{1}{3}} \mathrm{~d} t$

and it is invariant under affine transformations. The Cartan matrix now has the form

$\mathcal{C}(A)=\left(\begin{array}{cr}0 & 1 \\ -\left|\mathbf{x}^{\prime \prime} \wedge \mathbf{x}^{\prime \prime \prime}\right| & 0\end{array}\right)$

and its only non-constant entry is the affine curvature:

$\kappa \equiv\left|\mathbf{x}^{\prime \prime} \wedge \mathbf{x}^{\prime \prime \prime}\right|$

which is the second affine invariant function. The affine plane Frenet equations are

$\begin{aligned} \mathbf{x}^{\prime} & =\mathbf{a}_{1} \\ \mathbf{a}_{1}^{\prime} & =\mathbf{a}_{2} \\ \mathbf{a}_{2}^{\prime} & =-\kappa \mathbf{a}_{1},\end{aligned}$

whence $\mathbf{x}^{\prime \prime \prime}=-\kappa \mathbf{a}_{1}$.

The expression of the affine curvature in terms of a general parameter $t$ is

$$
\begin{aligned}
\kappa(t)= & -\frac{5}{9}|\dot{\mathbf{x}} \wedge \ddot{\mathbf{x}}|^{-\frac{8}{3}}|\dot{\mathbf{x}} \wedge \dddot{\mathbf{x}}|^{2}+\frac{4}{3}|\dot{\mathbf{x}} \wedge \ddot{\mathbf{x}}|^{-\frac{5}{3}}|\dot{\mathbf{x}} \wedge \dddot{\mathbf{x}}| \\
& +\frac{1}{3}|\dot{\mathbf{x}} \wedge \ddot{\mathbf{x}}|^{-\frac{5}{3}}|\dot{\mathbf{x}} \wedge \dddot{\mathbf{x}}|,
\end{aligned}
$$

which shows that affine curvature is a fourth order differential function even though only a third order derivative appears in Eq. (25). We should therefore assume that the curve has at least $C^{4}$ smoothness. The relation between affine and Euclidean curvatures is:

$\kappa=-R^{\frac{4}{3}}+1 / 2 \frac{\mathrm{d}^{2} R^{2 / 3}}{\mathrm{~d} s^{2}}$.

In addition, the affine curvature being a fourth order differential function has an important practical implication.

Implication: It is impractical to compute Eq. (27) numerically in real life applications, because the complexity of the expression and because the use of a high order derivative would cause any signal to drown in noise. Fortunately, though, a novel numerical method has recently been developed, which directly uses affine invariant quantities and altogether avoids expressions such as Eq. (27). This method is described in Sect. 6.2. 
Additional higher order differential invariant functions are obtained by differentiating the affine curvature w.r.t. to affine arc-length. This is an example of a general result regarding curves that evolve under the action of a Lie group.

In this paper we are interested only in the first of these additional functions, namely $\kappa^{\prime}(\sigma)$, which will be used later. One important result in which this function appears is:

Definition 2 (Guggenheimer 1977) A sextactic point on a $C^{4}$ curve is one where $\kappa^{\prime}(\sigma)=0$.

A closed convex $C^{4}$ curve has at least six sextactic points (for a proof see Guggenheimer 1977). Thus, when studying closed or almost smoothly closed curves traced by the hand, we might expect oscillations in the affine curvature function. Finally, one additional remark: at points where $\mathbf{x}^{\prime} \wedge \mathbf{x}^{\prime \prime}=$ 0 , such as inflection points, the affine frame is not defined. Hence, these inflection points could provide natural points for segmentation of a curve into strokes. Since, however, the affine frame is undefined at such points, the movement in their vicinity could be segmented into three strokes: two curved segments, before and after the inflection point, and a third straight segment for passing through the inflection point.

\subsection{Invariant representations of curves}

Above we described a curve moving frame structure. This was used to extract invariant functions of the curve associated with a Lie group. We will now travel this path in reverse and see how the Cartan matrix, which contains the invariant functions, acts locally on the moving frame to produce the original curve, modulo global group transformations. In addition, these invariant functions will be used to construct invariant representations of families of curves that are invariant under global actions of the group $G$.

Recall that the frame matrix $A(\sigma)$ is a parameterized element of the group, $G=S L(2)$, i.e., it forms a curve in the group. Its Cartan matrix $\mathcal{C}(A)$ is an element of the Lie algebra $\mathfrak{s l}(2)$. It, in turn, determines the local evolution of the frame, particularly that of the tangent vector. The parameterdependent tangent vector field can be integrated to reconstruct the original plane curve up to an initial position. Moreover, according to the following lemma, the Cartan matrix is invariant under a fixed global group transformation and, therefore, the reconstructed curve is determined only modulo group actions.

Lemma Given a parameterized frame matrix $A(\sigma) \in G$ and a fixed element $B \in G$, the Cartan matrix is invariant under the group action $B$ from the right: $\mathcal{C}(A B)=\mathcal{C}(A)$ (Guggenheimer 1977).

Result: The frame matrix function determines a curve modulo a fixed global transformation of the group of plane affine motions $S A(2)$.
The only non-constant entry in $\mathcal{C}(A)$ is the affine curvature $\kappa(\sigma)$ as a function of affine arc-length $\sigma$. Following the previous result, $\kappa(\sigma)$ itself determines a curve modulo the group action. Conversely, two curves can be obtained one from the other by a group action, and both have the same affine curvature function. The latter can therefore be thought of as a template for an infinite family of curves related by group actions.

There is another invariant representation of families of curves by the pair of functions $\kappa(\sigma)$ and $\kappa^{\prime}(\sigma)$. Since both are parameterized by $\sigma$, they determine a curve in the phase plane $\left(\kappa, \kappa^{\prime}\right)$, which is called the signature curve of the original curve in the plane, $\mathbf{A}^{2}$. Signature curves of hand traced trajectories are shown in Sect. 6.4.

Following the above discussion let us look at some specific example. A special case is when $\kappa$ is constant, because the Cartan matrix is then a fixed element in the Lie algebra and it generates a one-parameter subgroup in the group. The frame matrix can therefore be obtained directly as a matrix exponent function of the Cartan matrix, i.e.:

$$
A=\operatorname{EXP}\{\sigma \mathcal{C}(A)\}
$$

It can be shown (Guggenheimer 1977) that for constant affine curvature and for $\kappa \neq 0$ the affine matrix is:

$A(\sigma)\left(\begin{array}{lr}\cos \kappa^{\frac{1}{2}} \sigma & \kappa^{-\frac{1}{2}} \sin \kappa^{\frac{1}{2}} \sigma \\ -\kappa^{\frac{1}{2}} \sin \kappa^{\frac{1}{2}} \sigma & \cos \kappa^{\frac{1}{2}} \sigma\end{array}\right)$

and for $\kappa=0$

$A(\sigma)=\left(\begin{array}{ll}1 & \sigma \\ 0 & 1\end{array}\right)$

The curves with constant affine curvature functions are the conics (Guggenheimer 1977; Griffiths 1983). Given that the first row of $A$ is the tangent vector, namely velocity, integrating it for $\kappa=0$ gives the curve:

$\mathbf{x}(\sigma)-\mathbf{x}(0)=\left\{x_{1}(\sigma), x_{2}(\sigma)\right\}=\left\{\sigma, \frac{1}{2} \sigma^{2}\right\}$

which is a parabola with initial position $\mathbf{x}(0)$. Notice also that its signature curve is a fixed point at the origin of the phase plane. Section 5 is devoted to a discussion of parabolas as affine plane geodesics. Similarly, integrating the first row of $A$ for gives an ellipse where $\kappa>0$ :

$$
\begin{aligned}
& \mathbf{x}^{\prime}(\sigma)=(\cos \sqrt{\kappa} \sigma, 1 / \sqrt{\kappa} \sin \sqrt{\kappa} \sigma) \\
& \mathbf{x}(\sigma)=\text { constant }+\left(1 / \sqrt{\kappa} \sin \sqrt{\kappa} \sigma,-\frac{1}{\kappa} \cos \sqrt{\kappa} \sigma\right) .
\end{aligned}
$$

Finally, for the hyperbola where $\kappa<0$ :

$\mathbf{x}(\sigma)=$ constant $+\left(\sqrt{|\kappa|} \cosh \sqrt{|\kappa|} \sigma, \frac{1}{|\kappa|} \sinh \sqrt{|\kappa|} \sigma\right)$. 
We now show that the following theorem can be proven in finding the curves corresponding to the extremum of $\sigma=$ $\int k^{\frac{1}{3}} \mathrm{~d} s$ (Griffiths 1983):

Theorem 1 (Griffiths 1983) The solutions of the Euler-Lagrange equations associated with the above integral are plane conics with zero affine curvature. Hence, parabolas are the unimodular affine geodesics. It can be shown that parabolic segments are affine geodesics having zero affine curvature and maximal affine distance (Lopez de Lima and Montenegro 1999). Thus if we are given two points $P$ and $Q$ in the plane and two concurrent straight lines $m$ and $n$ passing through $P$ and $Q$, respectively, then the unique arc of parabola determined by this configuration is an extremal for the affine distance along variations by locally convex curves passing through $P$ and $Q$ and tangent to $m$ and $n$ at these points (Lopez de Lima and Montenegro 1999) See also Sect. 5 .

\subsection{Movement with constant affine speed}

Given the above mathematical tools (Sect. 3), it is almost trivial to show that the one-third power law is a result of movement at constant affine speed. From the definition of curvature (Eq. 12) we have

$v(t)=|\dot{\mathbf{x}} \wedge \ddot{\mathbf{x}}|^{\frac{1}{3}} r^{\frac{1}{3}}$,

where $r$ is the Euclidean radius of curvature and from the definition of affine velocity (Eq. 22) this is the same as

$v(t)=\dot{\sigma} r^{\frac{1}{3}}$

In any segment of a trajectory during which the motion has a constant equi-affine speed, $\dot{\sigma}=G$, the empirical one-third rule is thus observed.

\section{Local isochrony and speed scaling}

We now show that both the local isochrony principle and the speed scaling property of human movements are compatible with our suggestion that arm movements are generated with a piecewise constant equi-affine velocity. Dealing first with local isochrony, it has been argued that the dependence of the gain factor on the Euclidean length of the path is the most direct expression of isochrony, i.e., the rather weak dependence of the movement duration within a stroke or a motion segment on its length, is mostly due to the modulation of the velocity gain factor $G$-giving rise to local isochrony. In particular, Viviani and Cenzato (1985) have shown that for simple closed patterns (such as ellipses), the parameter $G$ is constant throughout the entire movement and is related to the Euclidean perimeter $P$ by a power law $G=G_{T} P^{\beta}\left(G_{T}\right.$ and $\beta$ both $\geq 0$ ), where $G_{T}$ depends only on the tempo selected for the motion. Viviani and Cenzato further characterized local isochrony for the drawing of a double ellipse (which is similar to the limaçon treated here, see Sect. 6 below). If in the case of a double ellipse, local isochrony is obeyed, then: $T_{L}=T_{S}$ where $T_{L}$ and $T_{S}$ are the movement durations for the large and small ellipses, respectively. This tendency for local isochrony is compatible with our affine differential analysis as follows. For a single ellipse the total equi-affine arc-length $\sigma$ can be expressed as: $\sigma=2 \pi^{2} A^{\frac{1}{3}}$ where $A$ is the ellipse's area. If the movement is at constant affine velocity, then $\sigma=G T$ where $T$ is the total movement duration. Thus, if we use the usual parametrization of the ellipse with semi-axes $a$ and $b$, namely, $\mathbf{X}(u)=(a \cos u, b \sin u)$, $0 \leq u \leq 2 \pi$, then the affine velocity is $\dot{\sigma}=(a b)^{\frac{1}{3}}$, the affine perimeter of the ellipse is: $\sigma=(a b)^{\frac{1}{3}} u$, and the affine curvature is $\kappa=(a b)^{-\frac{2}{3}}$. The relationship between the area of an ellipse, $A$ and its Euclidean perimeter, $P$, can be expressed as follows (see Viviani and Cenzato 1985): $A=\pi P^{2 / 3} \Omega(\epsilon)$ where $\Omega(\epsilon)=\left(\frac{\left(1-\epsilon^{2}\right)^{\frac{1}{2}}}{16 E^{2}\left(\epsilon^{2}\right)}\right)^{\frac{1}{3}}, E$ denoting the complete elliptic integral of the second kind and $\epsilon^{2}=1-b^{2} / a^{2}$, is the ellipse's eccentricity. Returning to the double ellipse, if the movement is performed at a constant equi-affine velocity which is equal to the velocity gain factor, $G$, this implies that $\sigma_{L} / G_{L}=\sigma_{S} / G_{S}$ where $\sigma_{L}$ and $\sigma_{S}$ are the equi-affine perimeters and $G_{L}$ and $G_{S}$ are the velocity gain factors for the large and small ellipses, respectively. Using the above expression for the equi-affine perimeter, we see that this implies that $G_{L} / G_{S}=A_{L}^{1 / 3} / A_{S}^{1 / 3}$ where $A_{L}$ and $A_{S}$ are the areas of the large and small ellipses, respectively. Hence, expressing the area of both ellipses in terms of their Euclidean perimeters, if the small and large ellipses have the same eccentricity, then the relationships between their velocity gain factors can be expressed as: $G_{L} / G_{S}=P_{L}^{2 / 3} / P_{S}^{2 / 3}$ where $P_{L}$ and $P_{S}$ are the Euclidean perimeters for the large and small ellipses, respectively. This, therefore, is compatible with the empirical observations described by Viviani and Cenzato (1985), where the velocity gain factor was found to vary with the ellipse's Euclidean perimeter, according to: $G=G_{T} P^{2 / 3}$, $G_{T}$ being some constant that was found to be only mildly dependent on the ellipse's eccentricity.

Another earlier observation which is compatible with our suggestion that curved movements are generated with a constant equi-affine velocity is the time scaling property of human movements (Hollerbach and Flash 1982). If the fast and slow movements follow the same paths while the velocities are simply scaled with speed, then: $\left(x^{\prime}(t), y^{\prime}(t)\right)=$ ( $x(\lambda t), y(\lambda t))$ where $\lambda=T / T^{\prime}$ is the scaling factor expressing the ratio between the slow and fast movement durations, $T$ and $T^{\prime}$, respectively. Since according to the equi-affine description $G=A k^{-2 / 3}=V k^{1 / 3}$ and $\sigma=\int k^{\frac{1}{3}} \mathrm{~d} s=$ 
$\int G \mathrm{~d} t$, this implies that $V^{\prime}=\lambda V ; k^{\prime}(t)=k(\lambda t) ; G^{\prime}=$ $\lambda G$, where $V^{\prime}$ and $V$ are the Euclidean velocities and $k$ and $k^{\prime}$ are the Euclidean curvatures for the fast and slow movements, respectively. Consequently: $\int \mathrm{d} \sigma^{\prime}=\int \mathrm{d} \sigma$, i.e., the equi-affine arc-lengths are equal for the two paths and speed scaling, therefore, is equivalent to keeping the equi-affine arc length invariant under speed changes.

\section{Parabolic segments}

Here we focus on the description of parabolic pieces as simple segments that can be produced by moving between a pair of initial and final locations with prescribed movement directions at these endpoints. We provide an analytic solution for parabolic interpolation between such pairs of line elements within equi-affine plane geometry. The desired curves are the geodesics of equi-affine plane geometry, namely parabolic arcs, which generalize the connection of points by straight lines in Euclidean geometry and are invariant under the larger group of equi-affine transformations, $S A(2)$. The description of parabolic segments is of particular interest since they are repeatedly observed in human and monkey movements (see Sect. 7).

The problem discussed here is as follows: find a smooth curve that passes through a specified pair of end-points with specified directions (tangents) at these end-points. In finding such an interpolating curve, the premise is that it should be as smooth as possible, without points of unnecessarily high curvature. In vision research many studies have focused on the problem of minimizing Euclidean curvature along the interpolating curve, cast as the variational problem of minimizing the functional:

$\int k^{2} \mathrm{~d} s$

where $k$ is the usual Euclidean curvature and $\mathrm{d} s$, the Euclidean arc-length differential (Horn 1983). Scale invariant variations of Eq. (33) have also been used (Bruckstein and Netravali 1990), where the length of a curve is normalized. Similar functionals which represent the elastic energy of a flexible rod of fixed length have become popular:

$\int\left(\alpha k^{2}+\beta\right) \mathrm{d} s$

where $\alpha, \beta$ are constants. Solutions to Eq. (34) are therefore called elastica and the long history of their investigation is briefly recounted in Mumford (1994). Elastica are expressed using elliptic integrals (Griffiths 1983; Mumford 1994) for which numerical procedures and approximations (Sharon et al. 2000) are needed in practice.
Here we address the problem of interpolating between a pair of prescribed line elements that have a natural mathematical representation in affine geometry, leading to the formulation of the interpolation problem as a search for the curve which is an extremum of the affine arc-length integral:

$\int \mathrm{d} \sigma$.

The solution to this problem is the geodesic curve of equiaffine geometry and a natural generalization of the Euclidean straight line.

Below we provide a proof of the maximization of the equiaffine arc-length by parabolas, which also demonstrates that they are the equi-affine plane geodesics. Then we describe the parabolic interpolation scheme. A pair of contact elements determines a pencil of conics which contains one parabola. This pencil of conics is first constructed and the equation of the unique interpolating parabola is then derived using classical analytical geometry.

\subsection{Affine plane geodesics}

As was mentioned above (Sect. 3), the pure affine structure does not possess a measure of distance or length; instead, volume-i.e., area, in the plane-serves as a fundamental quantity. Nevertheless, a rudimentary form of length measurement exists as the ratio between segments of parallel lines. This property will be used in Theorem 2 .

In analogy to Euclidean geodesics, we seek curves that are extremals of the affine arc-length integral:

$\int \mathrm{d} \sigma=\int k^{\frac{1}{3}} \mathrm{~d} s$,

where the right-hand side is expressed in terms of the Euclidean curvature and arc-length. A variational approach to this functional is somewhat tricky and requires care in dealing with boundary conditions (Griffiths 1983). If the end-point conditions are specified as line elements, then among all interpolating convex curves there is a unique parabola and it maximizes affine arc-length Eq. (36). However, this is not the only extremum; for the same end-point conditions one can construct infinitely many interpolating curves that asymptotically tend to polygons, and they have arbitrarily small (positive) affine arc-length. The reason is the following: a vertex of the polygon can be considered as a circular arc of vanishing radius of curvature. The affine arc-length of this arc is dominated by its length which tends to zero. The straight segments have finite length but vanishing curvature, so their affine arc-length also vanishes. Hence any polygonal curve is a minimum of Eq. (36). In order to avoid this difficulty of picking the correct extremum in a direct variational treatment we instead present a constructive proof. 


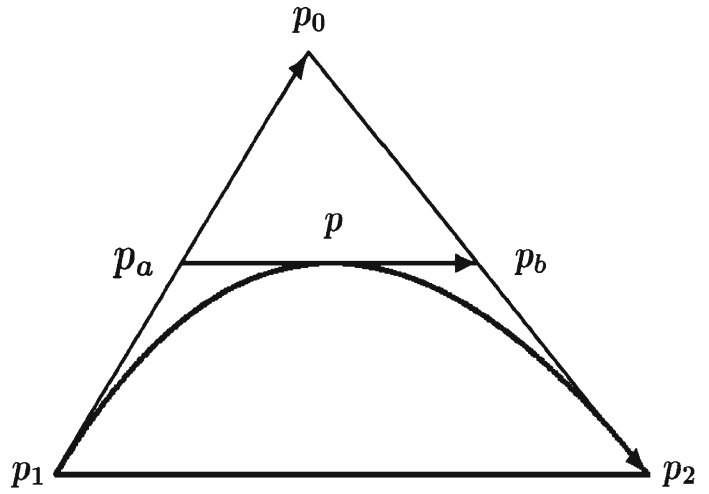

Fig. 2 The support triangle $\Delta\left(p_{1}, p_{0}, p_{2}\right)$ of a short $\operatorname{arc} \Gamma\left(p_{1}, p_{2}\right)$ is determined by the end-points $p_{1}, p_{2}$ and by the intersection $p_{0}$ of the given tangents at the end-points. The sides of the triangle are $p_{1} p_{2}, p_{1} p_{0}$ and $p_{0} p_{2}$. Every point $p \in \Gamma\left(p_{1}, p_{2}\right)$, splits the arc into two smaller ones. The tangent to $p$ cuts the support triangle at points $p_{a}$ and $p_{b}$. The two small arcs have support triangles $\triangle\left(p_{1}, p_{a}, p\right)$ and $\triangle\left(p, p_{b}, p_{2}\right)$

In such a construction, the idea is to concentrate the change in direction of the curve, i.e., high curvature regions, into arbitrarily short sections. For example, these sections can be made circular, where the leading term of the affine arclength tends to zero as the $2 / 3$ power of the radius of the circle. At the sections of the curve which are almost straight, the length is bounded, the curvature is arbitrarily small, and the affine arc-length therefore tends to zero. Only locally convex curves were considered in Sect. 2, i.e., those without inflection points. We further restrict the discussion to short segments of curves according to the following definitions (Olver et al. 1996):

Definition 3 A locally convex arc $\Gamma\left(p_{1}, p_{2}\right)$, together with its endpoints $p_{1}$ and $p_{2}$, is called a short arc if no two tangent lines to it are parallel. The equivalent statement in Euclidean geometry is that the total turning angle of the tangent to the curve from $p_{1}$ to $p_{2}$ is less than $\pi$.

Definition 4 Given a short arc $\Gamma\left(p_{1}, p_{2}\right)$, its support triangle $\triangle\left(p_{1}, p_{0}, p_{2}\right)$ is prescribed by its vertices: $p_{1}, p_{2}$, and the point $p_{0}$ where the tangents to $p_{1}$ and $p_{2}$ intersect. Its sides are the line $p_{1} p_{2}$ connecting the two end-points and the two tangents to these points, as depicted in Fig. 2.

We seek an affine invariant distance function between line elements which corresponds to the usual affine arc-length integral along curves. Since area is the fundamental affine invariant quantity, the support triangle of a short arc will be the basis for defining this affine distance. We denote by $\mathbf{A}\left(p_{1}, p_{2}\right)$ the area of the support triangle of the short arc $\Gamma\left(p_{1}, p_{2}\right)$.

Definition 5 The affine distance between two line elements $\left(p_{1}, \mathbf{a}_{1}\right)$ and $\left(p_{2}, \mathbf{a}_{2}\right)$ is

$\mathbf{d}\left(p_{1}, p_{2}\right) \equiv 2 \sqrt[3]{\mathbf{A}\left(p_{1}, p_{2}\right)}$.
In order to investigate the affine distance, we use the following construction. Let $\Gamma\left(p_{1}, p_{2}\right)$ be a short arc with a support triangle $\triangle\left(p_{1}, p_{0}, p_{2}\right)$, and let $p \in \Gamma\left(p_{1}, p_{2}\right)$ be an interior point whose tangent intersects the tangents $p_{1} p_{0}$ and $p_{0} p_{2}$ at $p_{a}$ and $p_{b}$ respectively. The support triangles of the $\operatorname{arcs} \Gamma\left(p_{1}, p\right)$ and $\Gamma\left(p, p_{2}\right)$ are thus $\Delta\left(p_{1}, p_{a}, p\right)$ and $\triangle\left(p, p_{b}, p_{2}\right)$ (see Fig. 2).

Theorem 2 (Blaschke 1923; Calabi et al. 1996)

Part 1: The affine anti-triangle inequality Given the construction above, the following inequality holds:

$\mathbf{d}\left(p_{1}, p\right)+\mathbf{d}\left(p, p_{2}\right) \leq \mathbf{d}\left(p_{1}, p_{2}\right)$.

Part 2: Equality is attained by parabolas and the condition for equality in Eq. (38) is

$\frac{p_{1} p_{a}}{p_{1} p_{0}}=\frac{p_{0} p_{b}}{p_{0} p_{2}}=\frac{p_{a} p}{p_{a} p_{b}}$,

where the ability to measure the ratio of parallel lines in the affine plane is used.

Remark: Interestingly, the equality condition (39) corresponds to the construction algorithm of quadratic de Casteljau-Bézier curves (Farin 1993), which are indeed parabolas; the control polygon of the algorithm is the support triangle $\Delta\left(p_{1}, p_{0}, p_{2}\right)$.

Theorem 2 provides one desired property of a distance function, namely additivity: given a pair of line elements, their interpolating parabola can be split at any point, and the corresponding affine distances add up to the original one. However, to fully justify Definition 5, we have yet to show that it is compatible with the usual affine arc-length. We use the Taylor expansion of a parabola in a parameter $\sigma$ :

$\mathbf{x}(\sigma)=\mathbf{x}_{0}+\sigma \dot{\mathbf{x}}_{0}+\sigma^{2} \frac{1}{2} \ddot{\mathbf{x}}_{0}$

where $\mathbf{x}_{0}=\mathbf{x}\left(t_{0}\right)$ is the initial point.

Lemma (Blaschke 1923) The affine arc-length of a parabolic arc is related to the area of its support triangle as follows:

$\mathbf{A}\left(\mathbf{x}_{0}, \mathbf{x}_{1}\right)=\frac{1}{8}\left|\dot{\mathbf{x}}_{0} \wedge \ddot{\mathbf{x}}_{0}\right| \sigma_{1}^{3}$

where $\sigma_{1}$ is the value of $\sigma$ at point $\mathbf{x}_{1}$, and therefore

$\mathbf{d}\left(\mathbf{x}_{0}, \mathbf{x}_{1}\right)=\left|\dot{\mathbf{x}}_{0} \wedge \ddot{\mathbf{x}}_{0}\right|^{\frac{1}{3}} \sigma_{1}$.

The right-hand side is proportional to the affine arc-length of the parabola, with the normalization factor determined 
at the initial point. If we choose the particular parametrization $\sigma$ (according to Eq. 21) then the affine distance (37) coincides with the affine arc-length, as required. This last result, together with the additivity of the affine arc-length along parabolas, completes the demonstration that the affine distance is well defined.

Finally, we extend the above to a general interpolating short arc between affine elements. Such an arc can be partitioned into segments. By inequality (38), the sum of the affine distances along these segments is a non-increasing function under refinement of the partition. Since the distances are positive, the series of sums is bounded from below and, therefore, it converges. For each segment, the affine distance approximates the affine arc-length, according to Eq. (42). With increasing refinement the sum of distances converges to the Riemann integral defining the affine arc-length along a curve. This proves the following:

Theorem 3 Parabolic arcs maximize the affine arc-length among all short arcs interpolating a pair of line elements (Olver et al. 1996). For the parabola the affine arc-length also equals the affine distance.

As a numerical example, we compare the affine arc-length of a parabolic arc and a quarter-circular arc, both having the same support triangle (whose vertices are located at $(0,0)$, $(0,1 / 2)$ and $(1 / 2,0)$ with the tangents to the endpoints coinciding with the principal axes. The equation of this parabola, as parameterized by affine arc-length, is $\left\{\frac{1}{2} \sigma^{2}, \frac{1}{2}(1-\sigma)^{2}\right\}$, $\sigma \in[0,1]$; its non-parametric form is $y=x-\sqrt{2 x}+\frac{1}{2}$. Clearly, its affine arc-length equals 1 . The affine arc-length of the quarter-circle is:

$\sigma($ circle $)=\int k^{\frac{1}{3}} \mathrm{~d} s=\sqrt[3]{2} 2 \pi r / 4=\sqrt[3]{2} \pi / 4 \approx 0.989$

which, as expected, is slightly smaller than 1 since the parabola maximizes the affine arc-length.

\subsection{Computing parabolic arcs}

When analyzing motion trajectories, it may be useful to construct the parabolic arc solution for a given pair of line elements. We compute the equation of a parabola which passes through two given line elements. We use the fact that these boundary conditions determine a one-parameter family of conics to which the sought parabola belongs. The two Cartesian coordinate variables are denoted here for convenience by $x$ and $y$. The equation of a conic is

$a x^{2}+2 b x y+c y^{2}+2 d x+2 e y+f=0$,

and the condition for it being a parabola is

$b^{2}-a c=0$.

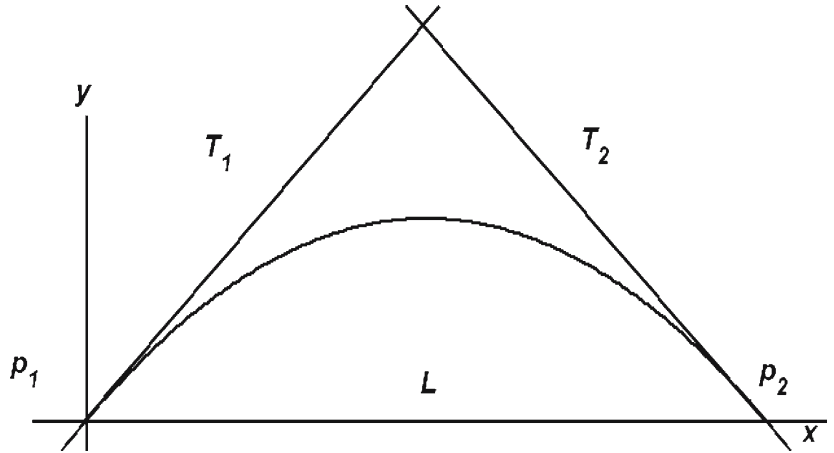

Fig. 3 The pencil of conics of double contact is determined by the two tangents $T_{1}$ and $T_{2}$ and the line $L$ connecting the two points of tangency $p_{1}$ and $p_{2}$

We need to find the coefficients $a$ through $f$ which satisfy the end conditions. In what follows we employ the standard notation for algebraic curves: the term " $F=0$ " denotes an algebraic plane curve, i.e., the zero level-set of a polynomial function $F$ in $x$ and $y$.

Definition 6 Given two distinct conics $C_{1}=0$ and $C_{2}=0$, a pencil of conics $\mathcal{C}$ is a family of conics depending on a parameter $\lambda$ (Spain 1957):

$\mathcal{C} \equiv C_{1}+\lambda C_{2}=0$.

In general, two conics have four points of intersection (not all necessarily real), so the pencil $\mathcal{C}$ comprises the conics passing through these four base points.

The product of two lines is a quadratic curve which is considered degenerate. The conics chosen to build $\mathcal{C}$ can therefore be line pairs, e.g., $C_{1}=T_{1} T_{2}$. A special case arises when the base points are divided into two pairs with the points of each pair coinciding, so that the two lines $T_{1}=0$ and $T_{2}=0$ pass through them.

Definition 7 If the four base points of a pencil "coalesce" in pairs to two points, then the conics of the pencil are said to have double contact.

This configuration is shown in Fig. 3: the line $T_{1}$ is tangent on the conics at point $p_{1}$, and $T_{2}$ is tangent at point $p_{2}$; the line $L$ connects these points.

Another degenerate conic in the pencil is the line $L$ taken twice, i.e. $L^{2}$. By substituting the two mentioned line pairs for the conics $C_{1}$ and $C_{2}$, we obtain the following representation of the pencil

$\mathcal{C} \equiv T_{1} T_{2}+\lambda L^{2}=0$.

This form suits our parabolic interpolation completion task because it can be constructed directly from the given boundary conditions. The next step is to calculate the value of $\lambda$ for 


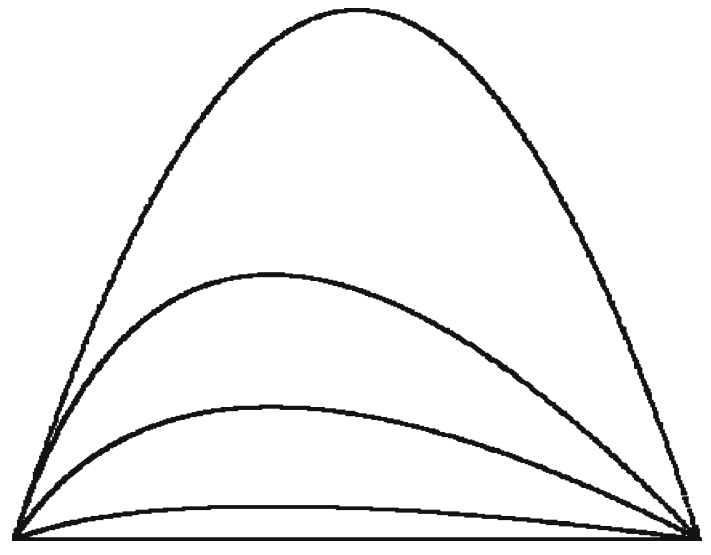

Fig. 4 Interpolating parabolic arcs between pairs of line elements of various orientations, covering the broad range of turning angles between $0^{\circ}$ and $152^{\circ}$

which Eq. (46) is a parabola. In order to simplify the calculation, the coordinate system is transformed (by translation and rotation) so that $L$ lies on the $x$-axis, and the axes-origin coincides with point $p_{1}$. The equations of the three lines reduce to

$$
\begin{aligned}
L & =y \\
T_{1} & =y+t_{1} x \\
T_{2} & =y+t_{2} x+s_{2}
\end{aligned}
$$

which are then substituted in the formula of the pencil of conics (Eq. 46). Comparing it with the general equation of a conic (Eq. 43) and by imposing the condition for a parabola (Eq. 44), the expression for $\lambda$ is obtained:

$\lambda=\frac{\left(t_{1}+t_{2}\right)^{2}}{4 t_{1} t_{2}}-1$.

Finally, by substituting the solution for $\lambda$ in Eq. (46), we find the explicit formula of the required parabola:

$\mathcal{P}=\left(t_{1} t_{2}\right) x^{2}+\frac{\left(t_{1}+t_{2}\right)^{2}}{4 t_{1} t_{2}} y^{2}+\left(t_{1}+t_{2}\right) x y+\left(s_{2} t_{1}\right) x+s_{2} y$.

Transforming back to the original image coordinates is straightforward (Spain 1957).

Figure 4 shows examples of interpolating parabolic arcs between pairs of line elements of various relative orientations covering a broad range of turning angles: $0^{\circ}-152^{\circ}$. These configurations are detailed in Table 1 where the slope of the line element is given relative to the connecting line $y=0$. When the turning angle between the line elements is zero, i.e., they lie on one straight line, the interpolating parabola flattens to become the line as desired. A turning angle of $180^{\circ}$ corresponds to the line elements being parallel.

We presented here an elegant analytic solution for interpolating between line-elements based on equi-affine plane
Table 1 A pair of line element configurations depicted in Fig. 4

\begin{tabular}{lll}
\hline $\begin{array}{l}\text { Left line element } \\
\text { Slope } t_{1}\end{array}$ & $\begin{array}{l}\text { Right line element } \\
\text { Slope } t_{2}\end{array}$ & $\begin{array}{l}\text { Turning angle } \\
\text { of curve }\left(^{\circ}\right)\end{array}$ \\
\hline 0 & 0 & 0 \\
$1 / 2$ & $-1 / 6$ & 36.1 \\
2 & $-2 / 3$ & 97.1 \\
4 & $-4 / 3$ & 129.1 \\
4 & -4 & 152 \\
\hline
\end{tabular}

geometry. The given data of the problem, namely the endpoint locations and the directions of the movements at these end-points, are naturally modeled as line elements in the affine plane. The interpolating curves are parabolas, which are the geodesic curves of the affine plane and they constitute a natural geometric extension of the concept of Euclidean geodesics. A straight line is the shortest path between any two points and its length determines the distance between them; the parabola maximizes the affine arc-length between a pair of line elements which, in turn, equals the affine distance between them; the respective curvature functions are zero along both curves. It is important to note that the affine arc-length is related to area and does not have an intuitive relation to usual Euclidean length. Its maximization by geodesics, as opposed to minimization in Euclidean geometry, should not be too surprising or hindering. The parabola we have derived is also the interpolating quadratic de Casteljau-Bézier curve. An important property of parabolic arcs is their invariance under equi-affine transformations: a parabolic arc remains a parabolic arc. The group of equi-affine motions, $S A(2)$, is larger than the group of Euclidean motions, $S E(2)$, and is highly relevant to motor control, as we show here.

\section{Affine analysis of human hand movements}

We now analyze several examples of continuous repetitive tracing of closed curves. The affine differential invariants $\sigma$ and $\kappa$ were derived for these movements and were used to examine movement segmentation and classification.

We begin by describing the experimental paradigms and methods used to record movement data, followed by a description of the numerical procedures used. Finally, we present our results.

\subsection{Experimental methods}

Three types of figures previously used in Viviani and Flash (1995) and in Richardson and Flash (2002) were investigated: the cloverleaf, the oblate limaçon ("double ellipse") and the symmetric and asymmetric lemniscates ("figure eight"). Subjects traced these figural forms in the horizontal plane. The drawings were generated according to given templates drawn 
on paper. Three versions of the figure eight were tested; all had the same total curve length of $48 \mathrm{~cm}$ and extended approximately $18 \mathrm{~cm}$ along the longitudinal axis, but the relative size of the two loops constituting the figure was varied. Similarly, three versions of the double ellipse were tested, with overall length varying between 60 and $75 \mathrm{~cm}$ and measuring about $20 \mathrm{~cm}$ along the longitudinal axis. The cloverleaf covered a square area of approximately $15 \mathrm{~cm}$ by $15 \mathrm{~cm}$.

The subjects, who were all right-handed, were asked to trace the curves freely and continuously according to the templates. The cloverleaf, the double ellipse and the larger loop of the lemniscate were all traced counterclockwise; the small loop of the lemniscate was traced clockwise. Recording hand movement began after several cycles of movement had been performed and the motion had stabilized. For details of the experimental set-up and paradigms see Viviani and Flash (1995). Coordinate data were smoothed using a Gaussian filter with a low-pass cutoff frequency of $4 \mathrm{~Hz}$. Velocity, acceleration and jerk of the coordinates of the hand trajectories were estimated using finite difference approximation.

\subsection{Affine invariant numerical calculations}

We now describe the numerical method for extracting the required functions associated with the affine moving frame from data obtained by measuring hand trajectories. This method was developed in Olver et al. (1996) and Calabi et al. (1998) in the context of computer vision. The quantities which we wish to calculate - the arc-length, the curvature, and derivatives of the latter by the former-are invariants of the special affine group, $S A(2)$.

\subsection{Affine differential properties of drawing movements}

For a transitive group action, a plane curve can be fully reconstructed (modulo group action) from a prescribed and finite collection of differential invariants (Cartan 1935; Faugeras 1993). A curve in a Euclidean plane can be fully reconstructed from its curvature $C$ and $\frac{\mathrm{d} C}{\mathrm{~d} s}$, i.e., from its Euclidean signature curve. It can be shown that any affine differential invariant is a function of $\kappa$ and its higher derivatives with respect to $\sigma$. Furthermore, any curve in the affine plane is uniquely prescribed by $\kappa$ and its $\frac{\mathrm{d} \kappa}{\mathrm{d} \sigma}$, i.e., its affine signature (Olver et al. 1996; Calabi et al. 1998). Thus, two smooth $\left(C^{5}\right)$ curves $c_{1}(\sigma)$ and $c_{2}(\sigma)$ can be mapped to each other by an affine transformation, $g$ belonging to $S A(2)$, and only if their signature curves are identical.

The relations between Euclidean and affine differential invariants are as follows:

$$
\begin{aligned}
\frac{\mathrm{d} \sigma}{\mathrm{d} s} & =C^{1 / 3}, \\
\kappa & =-R^{\frac{4}{3}}+1 / 2 \frac{\mathrm{d}^{2} R^{2 / 3}}{\mathrm{~d} s^{2}} .
\end{aligned}
$$

The construction of practical numerical approximations of differential invariants which depend on higher order derivatives of parameterized functions of the curve is a non-trivial problem. Here we used the finite approximation methods of Calabi et al. (1996).

An appropriate numerical approximation is needed for estimating the underlying functions when dealing with data in the form of discrete sampled points. Unless the numerical procedure is chosen with care, it may destroy the invariance under the action of the relevant group. In order to overcome this problem, the procedure used here is based on quantities that are "well-behaved" under the group action, namely joint invariants of the group, which are defined as follows:

Definition 8 If $G$ is a group of transformations acting on a space $X$, then a joint invariant $J$ is a function $J\left(x_{1}, x_{2}, \ldots, x_{k}\right)$ depending on $k$ points $x_{i} \in X$ and which does not change under the simultaneous action of a transformation $g \in G$ on the above points, i.e.,

$J\left(g x_{1}, g x_{2}, \ldots, g x_{k}\right)=J\left(x_{1}, x_{2}, \ldots, x_{k}\right)$.

Let us look at the familiar example of Euclidean motions in a plane, namely translations and rotations. The basic joint invariant in this case is the distance function between two points $\mathbf{d}\left(p_{1}, p_{2}\right)=\left\|p_{1}-p_{2}\right\|$ which is also the length of a vector in the displacement plane $V$. Together with the external product between two displacement vectors, they form a complete set of joint invariants, which means that every joint invariant of the Euclidean group is a function of these two invariants.

In the affine plane the fundamental joint invariant is the area of the parallelogram (or triangle) determined by three points $p_{i}=\left(x_{i}, y_{i}\right) \in \mathbf{A}^{2}$ :

$\left[p_{i}, p_{j}, p_{k}\right]=\left(p_{j}-p_{i}\right) \wedge\left(p_{k}-p_{i}\right)=\operatorname{det}\left(\begin{array}{ccc}x_{i} & y_{i} & 1 \\ x_{j} & y_{j} & 1 \\ x_{k} & y_{k} & 1\end{array}\right)$

which we denote in short by $[i j k]$. Every joint invariant of the affine group is a function of the above, in complete analogy to the Euclidean case. A useful four-point invariant is

$$
\begin{aligned}
{[i j k l] } & =\left[p_{i}, p_{j}, p_{k}, p_{l}\right]=\left(p_{i}-p_{j}\right) \wedge\left(p_{k}-p_{l}\right) \\
& =[i j l]-[i j k]
\end{aligned}
$$

which is the difference of two areas. For five points there are ten fundamental triangular areas, of which only five are independent. The required computation is based on such a five-point configuration and its associated areas,

As mentioned above, the two basic invariants of affine plane curves are the affine arc-length and the affine curvature. We wish to approximate these quantities using the joint invariants. The approximation is based on the unique hyperosculating conic curve which passes through any five given 
points, which in the present context are five consecutive sampled points of a trajectory traced by the hand. A conic section has constant affine curvature and is, therefore, taken as the approximation of the affine curvature at the central point out of the five. The canonical equation of a conic curve is

$A x^{2}+2 B x y+C y^{2}+2 D x+2 E y+F=0$,

and the affine curvature is then given by

$\kappa=\frac{S}{T^{2 / 3}}$

where $S$ and $T$ are functions of the coefficients:

$S=A C-B^{2}=\operatorname{det}\left(\begin{array}{cc}A & B \\ B & C\end{array}\right), \quad T=\operatorname{det}\left(\begin{array}{ccc}A & B & D \\ B & C & E \\ D & E & F\end{array}\right)$

The key fact is that $S$ and $T$ can be expressed as combinations of the ten fundamental areas determined by the five given points, the precise expressions of which are somewhat cumbersome and the interested reader can find them in the original work of Calabi et al. (1998) and Olver et al. (1996).

An approximation of the differential affine arc-length at point $i$ is obtained similarly. Finally, the derivative of the affine curvature by the arc-length is computed at point $i$ as a simple finite difference approximation:

$\kappa^{\prime}(\sigma)=\frac{\kappa_{i+1}-\kappa_{i-1}}{L_{i}}$

where $L_{i}$ is the approximate affine arc-length of the segment connecting point $i-1$ to point $i+1$.

\subsection{Results}

Most of the trajectories displayed here, unless is otherwise specified, constitute one cycle each, taken from continuous repetitive tracing of templates of closed figures. Also, in the current section, the term "differential equi-affine arclength" is used interchangeably with equi-affine velocity. Equi-affine curvature is denoted in the following plots by $\kappa$. When inspecting the results of the affine differential analysis of the movement data it should be noticed that the derivation of equi-affine variables involves the calculation of relatively high order derivatives. Moreover, movement data are quite noisy, both because of measurement noise and because of intrinsic neuromotor noise. Hence, the oscillations appearing in the plots of the equi-affine variables (see below) persisted even when other numerical methods for calculating those variables were used. These methods involved the use of Fourier series expansion or of spline approximation of the recorded movement data and the resulting approximated position data sets were then used to calculate the equi-affine velocity, curvature, and its derivative with respect to equiaffine arc-length. Hence, these oscillations are not simply an artifact due to numerical differentiation of a finite-length data window.

\subsubsection{The two-thirds power law}

The kinematic power law is demonstrated through the conventional presentation of Log-velocity versus $\log$ radius of curvature for the three figural forms analyzed here, the cloverleaf, Oblate Limaçon ("double-ellipse") and the figure eight (see Fig. 5 left column). The average slopes of the plots of $\log$ velocity versus $\log$ radius of curvature (marked in all figures by $\beta$ ) correspond to the exponents in the power law relationship between velocity and radius of curvature. The velocity gain factor, which is equal to the equi-affine velocity corresponds to the intercepts of these curves with the Log velocity axis. The cloverleaf is characterized by one segment (Fig. 5 top right panel). For the Oblate Limaçon (Fig. 5, middle right panel) two separate straight parts can be discerned in this plot, both with a very similar slope but shifted relative to each other and with a transition segment them. Each of the straight segments corresponds to one of the loops in the Limaçon with its own "gain factor" or equi-affine speed (see below). For the asymmetrical figure eight (Fig. 5, bottom right panel), again two straight segments with quite similar slopes and a transition segment between them characterize the Log-velocity versus Log radius of curvature plot.

\subsubsection{Cloverleaf}

The cloverleaf is a locally convex curve along its whole length (Fig. 6, upper left panel). The equi-affine speed shown for several repetitions of the drawing of the clover-leaf (Fig. 6, upper right panel) is fairly constant throughout with small fluctuations that lack any particular pattern, as seen in the plot for the drawing of one cycle of drawing (middle left panel). Consequently, the plot of the equi-affine arc-lengthintegrated affine speed - as a function of time (middle right panel) is a neat linear curve, implying that physical time is a scalar multiple of equi-affine arc-length. The one-third power law is clearly obeyed in tracing this figure. If equiaffine speed is the criterion, the complete figure can be taken as a single segment. Affine curvature of the single cloverleaf cycle is plotted as a function of time in Fig. 6 (bottom left panel). It is positive throughout most of the curve, similarly to the limaçon, without any orderly pattern. Locally, therefore, most of the cloverleaf looks like an elliptical arc, i.e., its hyperosculating conics are ellipses. The signature curve of the cloverleaf (drawn in Fig. 6, bottom right panel) seems to wind around several average values of equi-affine curvature. This phenomenon results from the time-dependent fluctuations seen in the affine curvature plot. 


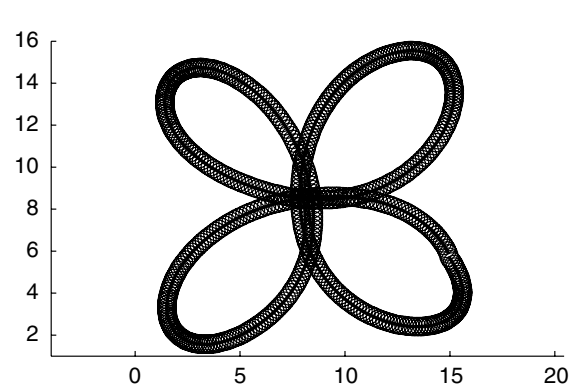

Cloverleaf

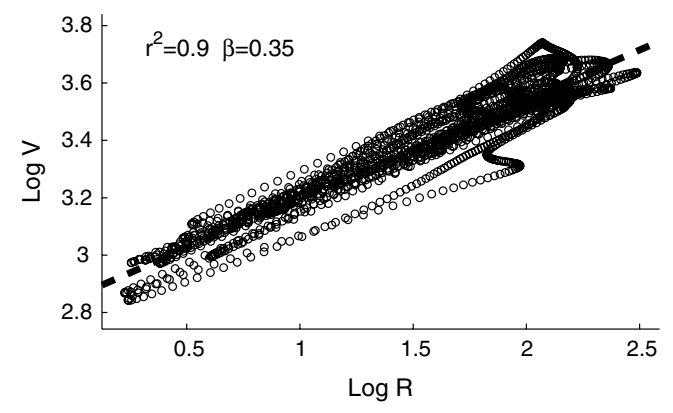

Oblate Limacon
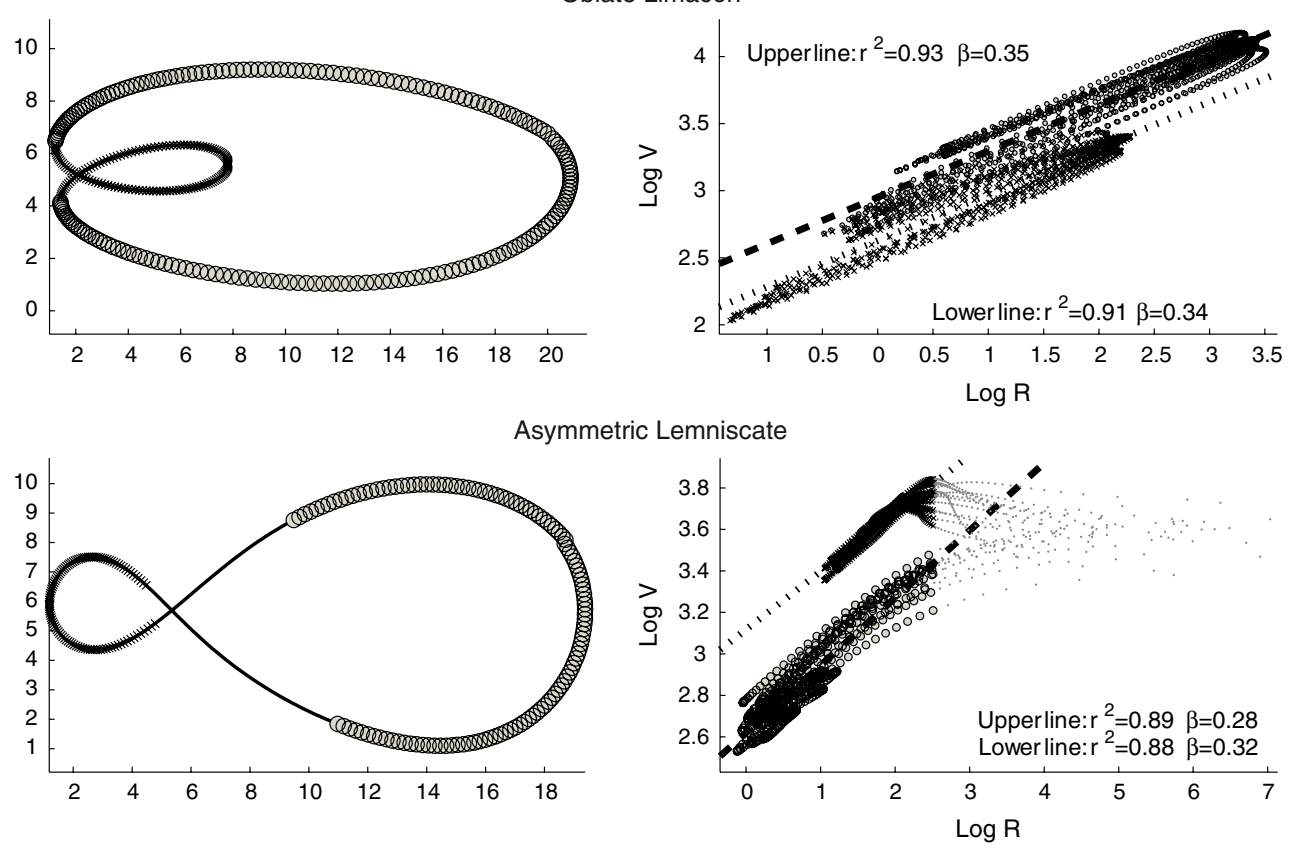

Fig. 5 Plots of log Euclidean speed $(V)$ versus log radius of curvature $(R)$ for three figures: cloverleaf (top row), oblate limaçon (middle row) and figure eight (bottom row). One cycle of drawing (left column) and several superimposed $\log V$ versus $\log R$ plots for several repetitions of the drawing (right column) for each figural form are shown. For the cloverleaf the data points of the drawing (upper left panel) are marked by circles and the corresponding points in the $\log V$ versus $\log R$ plot (upper right panel) are approximated by a single straight line $\left(r^{2}=0.9\right.$, $\beta=0.35$ ). For the oblate limaçon (middle row), the data points of the drawing (middle left panel) are marked by circles (for the exterior larger loop) and by crosses (for the interior smaller loop) and the corresponding data points in the $\log V$ versus $\log R$ plot (middle right panel) are similarly marked and are separately linearly approximated by two

straight lines $\left(r^{2}=0.93\right.$ and $\beta=0.35$ for the larger loop, upper line; $r^{2}=0.91 \beta=0.34$ for the smaller loop, lower line). For the asymmetric lemniscate (bottom row), data points with curvature smaller than $0.08 \mathrm{~cm}^{-1}$ are marked on the drawing (bottom left panel) by a solid line and on the plot of $\log V$ versus $\log R$ (bottom right panel) by gray dots. The rest of the drawing data points are divided into two according to the two loops of the asymmetric lemniscate, and are marked by circles and crosses, for the large and small loops, respectively (bottom left panel). The corresponding data points in the $\log V$ versus $\log R$ plot (bottom right panel) are again separately linearly approximated $\left(r^{2}=0.89\right.$, $\beta=0.28$ smaller loop, upper line; $r^{2}=0.88, \beta=0.32$, larger loop, lower line)

\subsubsection{Oblate Limaçon ("double ellipse”)}

A trace of a double ellipse with two loops of different size is shown in Fig. 7, upper left panel. Affine speed for the drawing of several cycles of the Limaçon, and for only one cycle are shown in Fig. 7, upper right panel and middle left panel, respectively. The equi-affine speed oscillates around an average value, more prominently than the minor fluctuations seen for the cloverleaf. This implies that, for the double ellipse,

the one-third power law is only approximately valid. A close look at the equi-affine speed within a single trace of the template (Fig. 7, middle left panel) shows that it varies gradually, being greatest at the apex of the larger loop of the figure and decreasing towards the smaller one. This pattern corresponds to the distinct values of the velocity gain factor in each of the loops, as reported in Viviani and Flash (1995) and as is demonstrated by the different intercepts of the upper versus the lower straight lines used in the linear approximations of the 
Fig. 6 Cloverleaf: typical results from the affine analysis of the drawings of a cloverleaf. Shown are one cycle of drawing (upper left panel), a plot of the affine speed versus time for several repetitions of the drawing of a cloverleaf (upper right panel), a "zoomed in" portion of the upper right panel, showing the affine speed versus time for one cycle of the cloverleaf (middle left panel) and a plot of the affine arc-length versus time for several repetitions of the drawing (middle right panel). Also shown is a plot of the affine curvature versus time for one cycle of the cloverleaf (bottom left panel) and the affine signature curve for one complete cycle of the cloverleaf (bottom right panel)
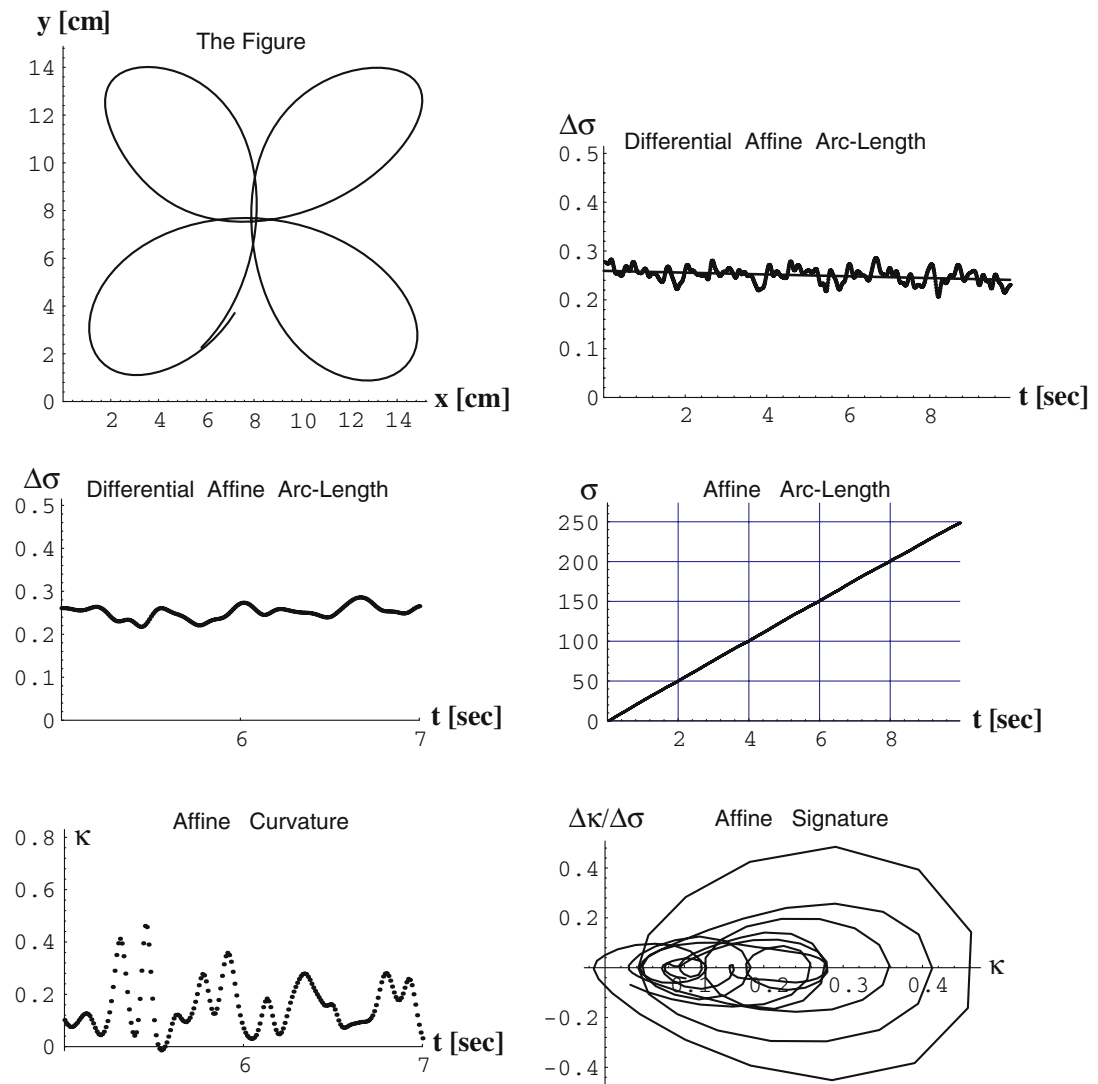

$\log$ velocity versus log radius of curvature data points for the the two loops of the double ellipse (Fig. 5, middle right panel). Also shown is a plot of the equi-affine arc-length versus time for several repetitions of the drawing of the double ellipse (Fig. 7 middle right panel). The equi-affine arc-length is roughly linearly related to time, exhibiting a minute effect of the described modulations in affine speed. The equi-affine curvature plot for a single cycle taken from a repetition of several cycles of the template is displayed in Fig. 7, bottom left panel; its signature curve appears to the right. Similarly to the cloverleaf, this curve is locally convex throughout, and its affine curvature is positive, meaning that locally it is like an ellipse. In contrast to the cloverleaf, equi-affine curvature of the double ellipse oscillates in a regular pattern as a function of time, having many clearly identifiable extrema (sextactic points). This is reminiscent of Theorem 3.3 on the existence of at least six such points on a smooth closed curve. Although the traced trajectory is not a smoothly closed curve, in this respect it behaves as if it were. The signature curve of the limaçon (Fig. 7, bottom right panel) exhibits an oscillatory pattern matching the one of affine curvature. Since the signature curve of an ellipse is a fixed point with constant affine curvature, the signature curve of the limaçon can be described as one which wobbles around two average ellipses.

\subsubsection{Lemniscate ("figure eight"): symmetric and asymmetric}

Lemniscates with loops either equal or unequal in size were examined. Single cycles of the traces of each of the two templates are shown in Figs. 8 and 9, respectively, together with the corresponding affine functions and signature curves.

In contrast to the two previous curves, equi-affine speed here has both positive and negative values. This results from $\mathrm{d} \sigma$ representing the oriented area of the local affine frame. The sign of the affine equi-speed reflects the orientation of the motion, positive values corresponding to segments of the curve traced counter-clockwise, and negative ones to clockwise tracing. The equi-affine speed of these two figures remains almost constant throughout each segment of a curve, thus the one-third power law provides an accurate description of the tracing of lemniscates. There is a sharp transition where equi-affine speed changes sign between adjacent segments. This zero-crossing of the equi-affine speed is due to passage through inflection points at which equi-affine functions are not well defined.

The total equi-affine arc-length of an ellipse reflects the area which it encloses. This also holds approximately true for convex figures which are not precise ellipses. Since the sizes 
Fig. 7 An Oblate Limaçon: typical results from the affine analysis of the drawings of an oblate limaçon. Shown are one cycle of drawing (upper left panel), a plot of the equi-affine speed versus time for several repetitions of the drawing of the limaçon (upper right panel), a "zoomed in" portion of the upper right panel, showing the equi-affine speed versus time for one complete cycle of the drawing (middle left panel) and a plot of the equi-affine arc-length versus time for several repetitions of the drawing (middle right panel). Also shown is a plot of the affine curvature versus time for one complete cycle of the oblate limaçon (bottom left panel) and the corresponding affine signature curve for one cycle of the drawing (bottom right panel)
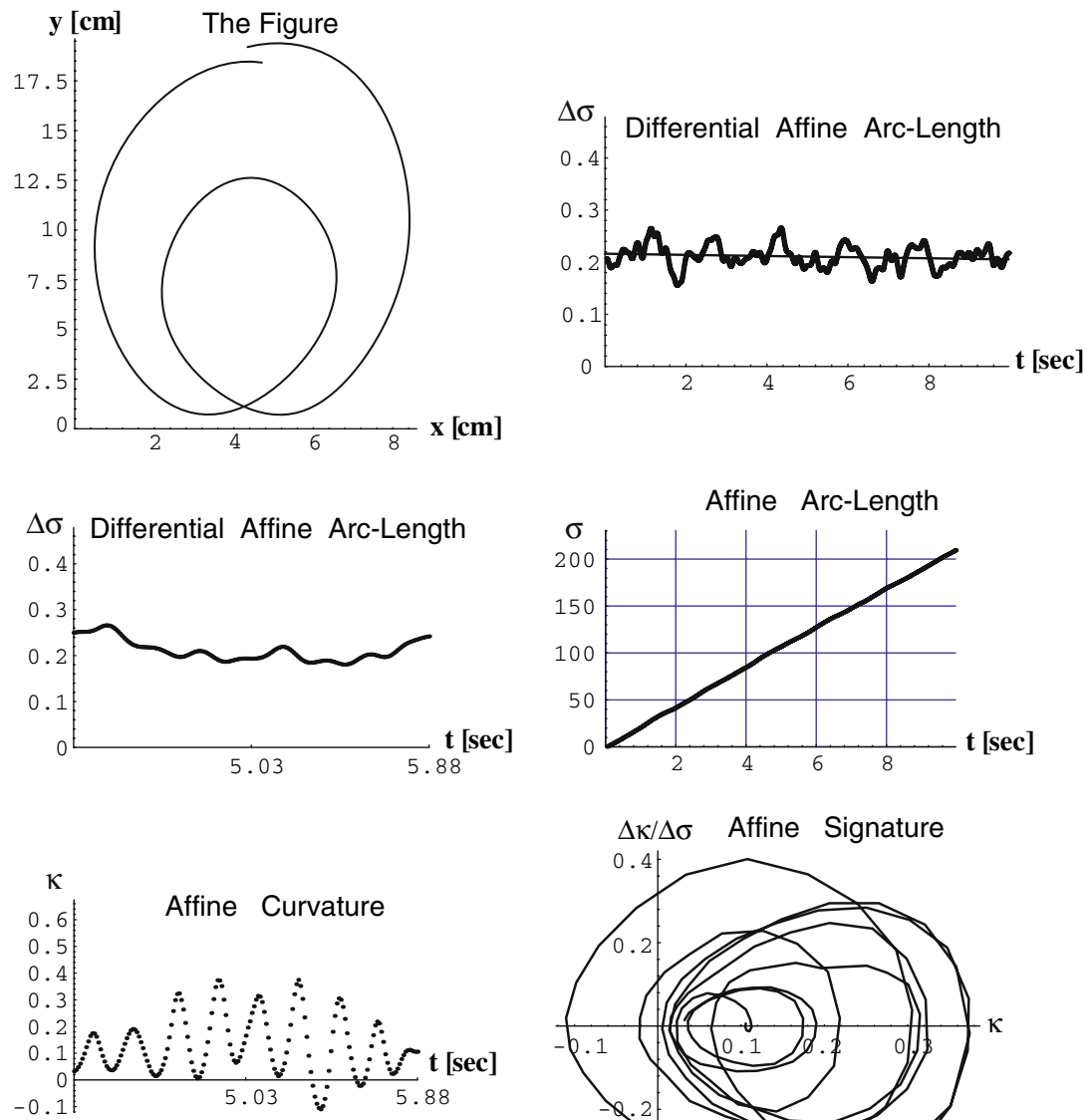

of the two loops of the lemniscate in Fig. 8 are more or less equal, the equi-affine arc-lengths along these loops are also equal (but opposite in sign), as can be seen in Fig. 8 (middle right panel). In the asymmetrical lemniscate the areas enclosed by the two loops differ, reflected by the positive "drift" of its affine arc-length in Fig. 9 (middle right panel). Notice that the magnitude of the equi-affine speed in the two loops is similar, but tracing the larger loop takes longer. This is the source for the larger equi-affine arc-length of that segment.

Compared with the limaçon, the equi-affine curvature of the lemniscate, as depicted in the bottom left panels of Figs. 8, and 9, exhibits a less orderly pattern. However, for the symmetrical lemniscate, equi-affine curvature fluctuates around an average positive value in the loop sections of the curve. In contrast to both the limaçon and cloverleaf, equi-affine curvature at some places drops sharply to large negative values. Similarly to equi-affine speed, these reflect the passage through inflection points in between the two loops of the figure. When coming out of a loop, and as the path straightens, the hyperosculating conic changes to hyperbolas, the conics with negative affine curvature. As the inflection point is approached, the affine curvature drops towards $-\infty$. This running-off of the affine curvature towards $-\infty$ is seen again in the signature curves of the lemniscates. Interestingly, the two signature curves in Figs. 8, and 9 are similar, implying that they could not be used to distinguish between lemniscates with varying ratio of loop sizes.

\section{Discussion}

We have applied a mathematical framework based on affine differential geometry to the analysis of $2 \mathrm{D}$ drawing movements. We show that the $2 / 3$ power law is compatible with the equi-affine velocity being piecewise constant and have developed a mathematical formalism for analyzing and characterizing human drawing movements based on the affine differential properties of these trajectories. Our analysis reveals that smooth hand trajectories are performed approximately with piecewise constant equi-affine velocity. Some trajectories, however, showed a patterned deviation from a constant equi-affine velocity, as in the tracing of the figure eight where the affine speed increased or decreased monotonously throughout each trajectory segment. 
Fig. 8 Symmetrical lemniscate: typical results from the affine analysis of the drawings of a symmetrical lemniscate. Shown are one cycle of drawing (upper left panel), a plot of the equi-affine speed versus time for several repetitions of the drawing of the symmetrical lemniscate (upper right panel), a "zoomed in" portion of the upper right panel, showing the equi-affine speed versus time for one complete cycle of the drawing (middle left panel) and a plot of the equi-affine arc-length versus time for several repetitions of the drawing (middle right panel). Also shown is a plot of the affine curvature versus time for one complete cycle of the symmetrical lemniscate (bottom left panel) and the corresponding affine signature curve for one cycle of the drawing (bottom right panel)

Fig. 9 Asymmetrical lemniscate: typical results from the affine analysis of the drawings of an asymmetrical lemniscate. Shown are one cycle of drawing (upper left panel), a plot of the equi-affine speed versus time for several repetitions of the drawing of the asymmetrical lemniscate (upper right panel), a "zoomed in" portion of the upper right panel, showing the equi-affine speed versus time for one complete cycle of the drawing (middle left panel) and a plot of the equi-affine arc-length versus time for several repetitions of the drawing (middle right panel). Also shown is a plot of the affine curvature versus time for one complete cycle of the asymmetrical lemniscate (bottom left panel) and the corresponding affine signature curve for one cycle of the drawing (bottom right panel)
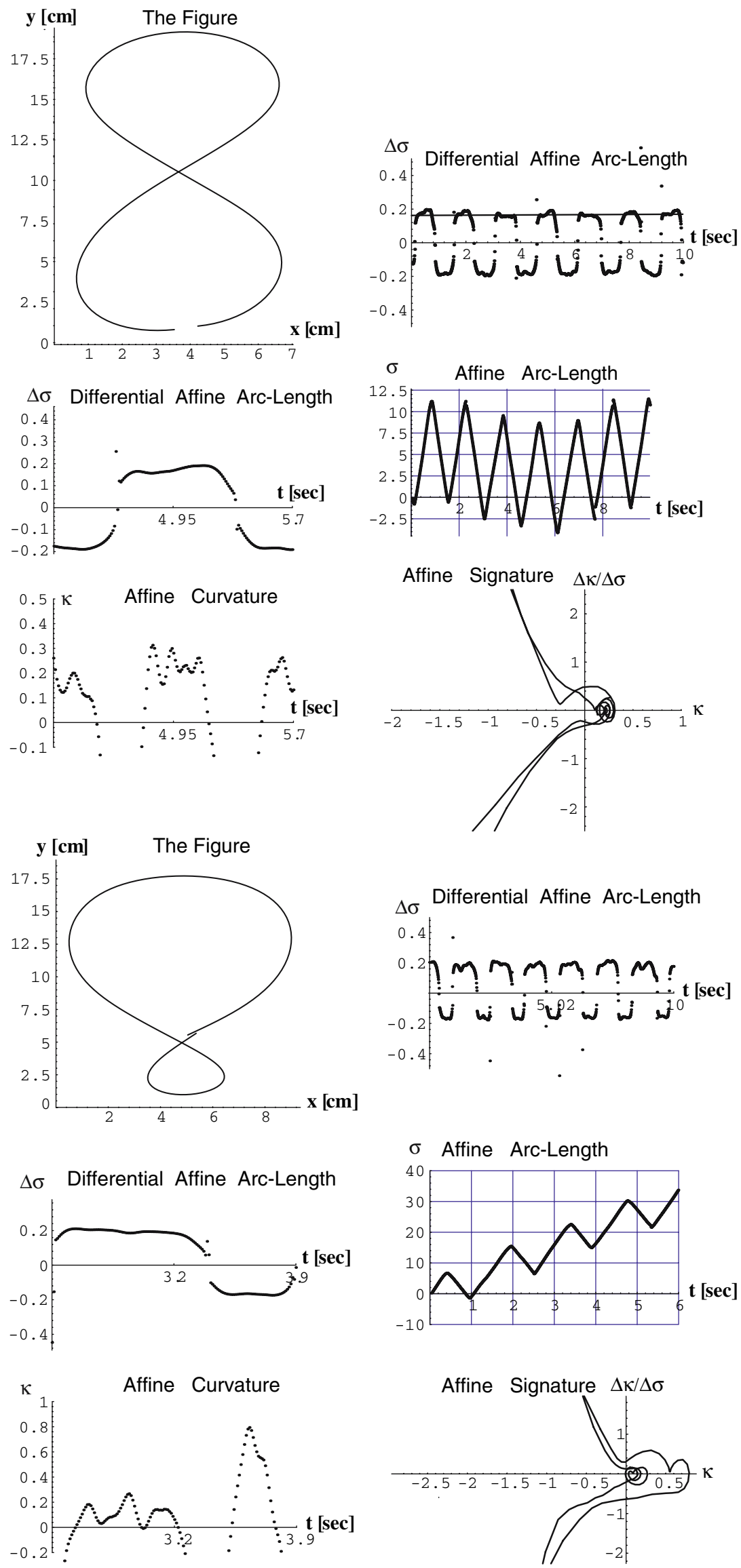
We also show that the affine description has several implications with respect to the temporal properties of human arm trajectories. These include the time scaling property of the movements, which is compatible with the total equi-affine arc-length being invariant under speed scaling. Furthermore, we show that local isochrony is consistent with the observed relation between the velocity gain factor and the Euclidean perimeter, as was previously observed (Viviani and Cenzato 1985; Viviani 1986).

7.1 The two third power law, affine analysis and neural dynamics

How can we explain the tendency observed during human and monkey arm trajectories to comply with the $2 / 3$ power law? A general model for the representation of movement variables in motor cortical areas involved movement encoding by using population vectors (Georolgopoulos et al. 1988; Schwartz 1992). Many neurophysiological studies have shown that population vectors calculated from the activities of neurons in the monkey motor cortex correspond to movement direction both in straight (Georolgopoulos et al. 1988) and in curved trajectories (Schwartz 1993, 1994). Extracting movement direction from the direction of the hand tangential velocity, monkeys' hand trajectories were successfully reconstructed by summing up the calculated time series of population vectors. The reconstructed trajectories preceded the actual motion by roughly $100-150 \mathrm{~ms}$, strengthening the interpretation of a causal relationship between the neuronal activity and hand motion.

Turning now to our issue of interest-the relation between population vector dynamics and the one-third power lawwe explore the implications of our affine analysis of arm movement generation for the neural coding of arm movements. Massey et al. (1992) suggested that the law could result from a constraint intrinsic to neural dynamics, which is expressed in the rotation velocity of a dynamically changing population vector. The important study by Schwartz (1994) established a direct link between population vector dynamics and the one-third power law by showing that the reconstructed neural trajectories obey the power law. In order to derive a quantitative constraint on neural dynamics, we take as our starting point the correspondence between the population vector $P_{v}$, and the tangential velocity vector of the hand $v$ (Schwartz 1994):

$P_{v} \sim \mathbf{v}(t)$

As shown in Sect. 3.5, the one-third power law is equivalent to movement at constant affine speed

$\dot{\sigma}=|\dot{\mathbf{x}} \wedge \ddot{\mathbf{x}}|^{\frac{1}{3}}=$ constant
In slightly different notation, this is the same as

$\mathbf{v} \times \dot{\mathbf{v}}=G$,

where " $\times$ " is the usual vector cross product and $G$ is a constant. By substituting relation (54) into (55) we have

$P_{v} \times \dot{P}_{v}=G$.

The left-hand side of this equation has the form of angular momentum of the population vector, although one should keep in mind that it is not the usual mechanical angular momentum, as $P_{v}$ does not represent a position variable. The equation expresses a conservation law which corresponds to the evolution of the moving frame of the hand's trajectory under area-preserving transformations, i.e., elements of $S L(2)$.

According to a well known principle of dynamical systems, conserved quantities usually reflect an underlying symmetry, expressed in the equations governing their dynamics. Conservation of angular velocity, in particular, reflects rotational symmetry; it results from the motion being determined by a central force field (Arnold 1989), i.e., one which depends only on the size ("norm") of the dynamical variable, and not on its direction. The general form of a Lagrangian with a central force field for the population vector is therefore

$L=\dot{P}_{v}^{2}+f\left(\left|P_{v}\right|^{2}\right)$,

where $f$ is an unspecified scalar function of the norm of the population vector.

We verify that if neural dynamics are governed by this Lagrangian, they result in the conservation of the angular velocity of the population vector, and consequently - the onethird power law. The Euler-Lagrange equation of (57) is

$\frac{\mathrm{d}}{\mathrm{d} t} \dot{P}_{v}-\frac{\partial f}{\partial\left(\left|P_{v}\right|^{2}\right)} P_{v}=0$.

Taking the vector product of $P_{v}$ with the Euler-Lagrange equation gives

$0=P_{v} \times \frac{\mathrm{d}}{\mathrm{d} t} \dot{P}_{v}+P_{v} \times P_{v} \frac{\partial f}{\partial\left(\left|P_{v}\right|^{2}\right)}=\frac{\mathrm{d}}{\mathrm{d} t}\left(P_{v} \times \dot{P}_{v}\right) ;$

thus recovering Eq. (56).

In fact, nowhere in the cortex is there any real single quantity corresponding to a population vector; rather, a population vector is a computational and conceptual tool serving as a condensed representation of distributed neuronal activity. The importance of Eq. (57) is, therefore, not in its explicit form, but that it may reflect a structural constraint inherent in the connectivity patterns and the dynamics of cortical neuron 
populations. In order to infer such constraints, neural network models of cortical areas are needed for which Eq. (57) can either restrict the model architecture or the parameter regime. One such model was designed specifically to reproduce hand movement generation (Lukashin et al. 1996). Another study showed how a network can lock onto and follow moving oriented stimuli (Ben-Yishai et al. 1997) or model hand trajectory generation (Ben-Yishai et al. 1995).

It is possible that variational principles such as aiming to generate maximally smooth movements lead to the planning of hand trajectories obeying the two-thirds power law (Richardson and Flash 2002). The minimization of other costs, such as variance (Harris and Wolpert 1998), also results in movements obeying this law. It is also possible that such variational principles are reflected in the dynamics of neural populations.

The above variational principle derived for the population vector is consistent with the power law and may be the underlying cause for the observed coupling between Euclidean curvature and velocity. Furthermore, the notion that each segment is performed at a constant equi-affine velocity points to an interesting analogy between a Kepler-like Law and the two-thirds power law by suggesting that the population vector sweeps equal areas within equal times, similarly to the position vector in Kepler's law.

When motor cortical activities were recorded while monkeys traced figure-eights (lemniscates) on a touch-sensitive computer monitor, population vectors constructed from these activities accurately and isomorphically represented the shape of the drawn figures (Schwartz and Moran 1999). The neural representation of the drawing was segmented in the same way as the movements and the two-thirds power law was evident in the neural correlate of the hand trajectory. These findings are therefore consistent with the two-thirds power law reflecting the underlying neural dynamics. This idea is also supported by smooth pursuit eye movements that apparently also obey the two-thirds power law (de'Sperati and Viviani 1997). Hence, this law may express some general principles of operation common to the different modules controlling the motor output of both the eye and the arm.

de'Sperati and Viviani (1997) further suggested that the neural events underlying directional coding may have their own dynamics: the larger the change in direction, the longer it takes to rotate the population vector. Thus, as Pellizzer et al. (1993) also hypothesized, the two-thirds power law may express a limitation on the rate at which higher control commands can modulate the activity of neural pools collectively coding the direction of a forthcoming movement. Moreover, since smooth pursuit eye movements also comply with this law, this may indicate that the same basic neural constraints apply across different neuronal pools.

Moran and Schwartz (1999a,b) and Schwartz and Moran (1999) have used drawing movements by monkeys to cal- culate the prediction interval describing the time interval between the population vector and the movement direction that best matched that direction. This interval increased in regions of high curvature that were also regions of low speed. The interval between the magnitude of the movement speed and the magnitude of population vectors varied directly with speed. Since the same population of cells codes for both direction and speed, the effective expression of this representation is reciprocal, occurring in different parts of each movement segment.

The population vectors in the Schwartz and Moran studies and in earlier studies by Georgopoulos and colleagues (Georolgopoulos et al. 1982, 1988) were represented in a laboratory-fixed Euclidean coordinate system. However, given our hypothesis that the affine metric is more appropriate for describing the neural coding of arm movements, using an affine moving coordinate system and representing the population vector in such a frame may lead to new and interesting findings on the behavior of the population vector and the prediction intervals. We have begun examining these possibilities by analyzing whether both neural activities and arm movements follow the power law during monkey drawing and whether affine differential variables subserve the representation of such movements (Polyakov et al. 2001).

\subsection{On the relation between motion perception and action}

Pollick and Sapiro (1997) have suggested that the power law may result from attempting to achieve affine invariance of the perceived movement under different visual viewing conditions. Related to this hypothesis, findings by Viviani and Stucchi $(1989,1992)$ indicate that the action and perceptual systems share similar invariants. The visual system is sensitive to the covariation between velocity and curvature which constrain biological motion. Thus, when this biological constraint is satisfied, a dot following a simple ellipse appears to move at a constant speed, although its velocity is highly nonuniform (Viviani and Stucchi 1989, 1992). More recently, Levit-Binnun et al. (2006) have confirmed this phenomenon using different elliptical trajectories with a large range of eccentricities, perimeters and different speeds. Furthermore, both de'Sperati and Viviani (1997) and Levit-Binnun et al. (2006) have demonstrated that the source of this behavior does not lie in the occulo-motor system, since the dynamic illusion is largely independent of eye movements.

To explain the findings of Viviani and Stucchi (1989, 1992), Pollick and Sapiro (1997) have pointed out that only a two-thirds power law is compatible with a constant affine velocity. Thus, two curves related to each other by an affine transformation are traveled with the same affine velocity, only if the affine velocity is governed by the $1 / 3$ power of Euclidean radius of curvature. Any other curve will not be affine invariant (Pollick and Sapiro 1997). 
Why is it advantageous that visual perception and motor production share a common representation and why should this representation be invariant under affine transformations? Viviani and Stucchi $(1989,1992)$ suggested that the observed similarities between visual perception and motor production arise from the influence of the active production of movements on visual perception. In contrast, Pollick and Sapiro (1997) argue that an affine perceptual encoding of visual form and movement has certain advantages and that the effects of visual perception on motor generation should not be excluded. For example, affine properties of shape are invariant to the relative orientation of the eye and the plane of a drawing motion. This may simplify both visual perception and motor production. Nevertheless, another plausible explanation for the reported similarities between the production of biological movements and their visual perception is that they may arise from similarities between the internal neural representations subserving both tasks and that these representations are best described in terms of equi-affine metrics (Levit-Binnun et al. 2006). Recently, we have conducted several studies aimed at relating the "macroscopic" type of constraints described here to neural activities recorded in cortical areas involved in motor and visuo-motor representations (Polyakov et al. 2001). Our findings from such studies will be described in a separate paper.

\subsection{Equi-affine analysis and motion primitives}

Another focus of the present analysis is on the hypothesis that complicated movements are constructed from a repertoire of basic motion primitives. We suggest that affine geometrical analysis can be used to further examine this hypothesis. Our analysis has already led to several interesting suggestions concerning movement segmentation and motor primitives. In particular, the equi-affine description has allowed us to define interesting relations between the equi-affine arc-length, Euclidean perimeter and the velocity gain factor which is equivalent to the equi-affine velocity. We have also developed mathematical tools that have allowed us to compare among the geometrical characteristics of different drawing movements, by examining the parameter-dependent profiles of these affine variables (e.g., affine arc-length and affine curvature) and the signature curves of these movements. While here, signature curves were only derived for complete figural forms, in ongoing studies, we have began to use these tools to examine to what extent different movement segments have been generated from a common template. Here, we want to emphasize, however, that our analysis of drawing movements suggests a possible geometrical nature for the underlying motion units and bears some relationship to earlier suggestions concerning what strokes may constitute such primitives. Soechting and Terzuolo (1987b) suggested that movement segments may be elliptical. As discussed here, ellipses are conics with constant positive affine curvature. However, the drawing movements analyzed here were not composed of elliptic segments. On the other hand, our recent analysis of monkey drawing movements has indicated that with practice monkeys tended to generate drawing movements that could be well modeled as a sequence of parabolic-like segments. Thus, based on the analysis of human hand trajectories that emerge following extensive practice (Sosnik et al. 2004), and affine differential analysis of monkey scribbling movements (Polyakov et al. 2001), it seems that drawing movements, in spite of their apparent continuity, are constructed of individual segments which are either straight or consist of curved, e.g., parabolic segments. Preliminary evidence supporting the notion that parabolic segments constitute an important primitive in human and monkey drawing movements, was discussed in Polyakov et al. (2001). Although any sufficiently smooth curve can be fitted with any specified precision by a sequence of parabolic segments, our observations have shown that monkey drawing movements are composed of a small number of relatively long parabolic segments. These findings will be described in greater detail in a forthcoming publication.

Finally, it is interesting to compare the equi-affine analysis presented here and the notions underlying optimization models. Previously it was shown that the experimentally observed exponents of a power law relationship between Euclidean velocity and curvature can be accounted for by assuming that the trajectories maximized motion smoothness (Viviani and Flash 1995; Richardson and Flash 2002). Thus, based on this result we have began to combine the analysis of hand trajectories using the affine differential geometry approach described here and trajectory formation models based on the optimization of kinematically defined objective functions (Polyakov et al. 2001). Another parameter required to describe a movement along a given path segment is the time needed to move along such segment. If, however, the movement is performed at a constant equi-affine speed, there is a linear relationship between the time needed to reach any point within the segment and the equi-affine length traveled till this point. We have demonstrated the relationship between the local isochrony principle and the equi-affine description. Further theoretical developments and the testing of their validity based on experimental data will be discussed in a future manuscript.

Given the success of the affine analysis in accounting for a large number of geometrical and temporal features of hand trajectories, we believe that our analysis of hand trajectories in terms of the underlying geometry does shed new light on fundamental questions such as the relation between motion perception and production, the underlying motor primitives, and the metrics that may subserve internal motor and visuomotor representations. 
Acknowledgments This research was supported in part by the German-Israeli Project Cooperation (DIP), by a grant from the Human Frontier Science Project (HFSP) and by the Moross Laboratory at the Weizmann Institute of Science. Tamar Flash is an incumbent of the Dr. Hymie Moross Professorial chair.

\section{References}

Arnold V (1989) Mathematical methods of Classical Mechanics, Volume 60 of Graduate Texts in Mathematics. Springer, Heidelberg

Atkeson CG, Hollerbach JM (1985) Kinematic features of unrestrained vertical arm movements. J Neurosci 5:2318-2330

Ben-Yishai R, Bar-Or R, Sompolinsky H (1997) Theory of orientation tuning in visual cortex: computation by a continuum of attractors. Proc Nat Acad Sci USA 92:3844-3848

Ben-Yishai R, Hansel D, Sompolinsky H (1995) Traveling waves and the coding of movement in a cortical network module. Israel J Med Sci 31:772

Berthier NE (1996) Learning to reach: a mathematical model. Develop Psychol 32(5):811-823

Blaschke W (1923) Vorlesungen uber differentialgeometrie vol. 2. Springer, Heidelberg

Bruckstein F, Netravali G (1990) On minimal energy trajectories. Comput Vis Graph Image Proces 49:283-296

Calabi E, Olver PJ, Tannenbaum A (1996) Affine geometr, curve flows, and invariant numerical approximations. Adv in Math 124:154-196

Calabi E, Olver PJ, Shakiban C, Tannenbaum A, Haker S (1998) Differential and numerically invariant signature curves applied to object recognition. Int J Comput Vis 26:107-135

Cartan E (1935) La methode du rep"ere mobile, la theorie des groupes continus, et les espaces generalisees. Exposes de Geometrie vol 5. Hermann, Paris

Conlon L (1993) Differential manifolds: a first course. Birkhauser

de'Sperati C, Stucchi N (1995) Visual tuning to kinematics of biological motion: the role of eye movements. J Neurosci 17:3932-3945

de'Sperati C, Viviani P (1997) The relationship between curvature and velocity in two-dimensional smooth pursuit eye movements. J Neurosci 17:3932-3945

Edelman S, Flash T (1987) A model of handwriting. Biol Cybern 57:2536

Farin G (1993) Curves and surfaces for computer aided design: a practical guide. Academic Press Professional, Inc.

Fasse DE (1992) On the use and representation of sensory information of the arm by robots and humans. Ph.D. thesis, Dept. of Mecahnical Engineering, MIT

Faugeras O (1993) Cartan's moving frame method and its application to the geometry and evolution of curves in the euclidian, affine and projective planes. Technical report, INRIA Technical Report, TR2053

Flash T, Gurevich I (1997) Arm trajectory generation and stiffness control during motor adaptation to external loads. In: Morasso PG, Sanguinetti V (eds) Self-organization, computational maps and motor control. Elsevier pp. 423-482

Flash T, Handzel AA (1996) Affine differential geometry of human arm trajectories. Abstr Soc Neurosci (in press)

Flash T, Henis E (1991) Arm trajectory modification during reaching towards visual targets. J CogNeurosci 3:220-230

Flash T, Hochner B (2005) Motor primitives in bertebrates and invertebrates. Current Opinion Neurobiol 15:660-666

Flash T, Hogan N (1987) The coordination of arm movement: an experimantally confirmed mathematical model. J Neurosci 5:1688-1703

Flash T, Sejnowski TJ (2001) Computational approaches to motor control. Current Opinion Neurobiol 11:655-662
Georolgopoulos AP, Kalaska JF, Caminiti R, Massey JT (1982) On the relations between the direction of two-dimensional arm movements and cell discharge in primate motor cortex. J Neurosci 2:1527-1537

Georolgopoulos AP, Kettner RE, Schwartz AB (1988) Primate motor cortex and free arm vovements to visual targets in three-dimensional space. ii. coding of the direction of movement by a neuronal population. J Neurosci 8:2928-2937

Gribble PL, Ostry DJ (1996) Origins of the power law relation between movement velocity and curvature: modeling the effects of muscle mechanics and limb dynamics. J Neurophysiol 76:2853-2860

Griffiths PA (1983) Exterior differential systems and the calculus of variations, vol. 25. Progress in Mathematics

Guggenheimer HW (1977) Differential geomtry. Dover

Harris CM, Wolpert DM (1998) Signal-dependent noise determines motor planning. Nature 394:780-784

Hollerbach JM, Flash T (1982) Dynamic interactions between limb segments during planar arm movement. Biol Cybern 44:67-77

Horn B (1983) The curve of least energy. ACM Trans Math Softw 9(4):441-460

Krebs HI, Aisen ML, Volpe BT, Hogan N (1999) Quantization of continuous arm movements in humans with brain injury. Proc Nat Acad Sci, USA, 96:4645-4649

Lacquaniti F (1989) Central representations of human limb movement as revealed by studies of drawing and handwriting. Trends Neurosci 12:287-291

Lacquaniti F, Terzuolo C, Viviani P (1983) The law relating the kinematic and figural aspects of drawing movements. Acta Psychol $54: 115-130$

Levit-Binnun N, Schectman E, Flash T (2006) On the similarities between the perception and production of elliptical trajectories. Exp. Brain Res 172:533-555

Lopez de Lima L, Montenegro J (1999) Classification of solitons for the affine curvature flow. Comm Analyt Geom 7:731-753

Lukashin AV, Amirikian BR, Mozhaev VL, Wilcox GL, Georgopoulos AP (1996) Modeling motor cortical operations by an attractor network of stochastic neurons. Biol Cybern 74:255-261

Massey JT, Lurito JT, Pellizzer G, Georgopoulos AP (1992) Threedimensional drawings in isometric conditions:relation between geomtry and kinematics. Exp Brain Res 88:685-690

Milner TE (1992) A model for the generation of movements requiring endpoint precision. Neuroscience 49:487-496

Moran DW, Schwartz AB (1999a) Motor cortical activity during drawing movements: Population representation during spiral drawing. $\mathrm{J}$ Neurophysiol 82:2693-2704

Moran DW, Schwartz AB (1999b) Motor cortical representation of speed and direction during reaching. J Neurophysiol 82:2676-2692

Mumford D (1994) Elastica and computer vision. Algeb Geom Appl 491-506

Mussa-Ivaldi FA, Solla SA (2004) Neural primitives for motion control. IEEE J Oceanic Eng 29:640-650

Olver PJ, Calabi E, Tannenbaum A (1996) Affine geometry, curve flows, and invariant numerical approximations. Adv Math 124:154-196

Olver PJ (1993) Applications of lie groups to differential equations. Springer, Heidelberg

Olver PJ, Sapiro G, Tannenbaum A (1994) Differential invariant signatures and flows in computer vision: a symmetry group approach. In: Ter Haar Romeny BM (ed) Geometry-driven diffusion in computer vision. Kluwer pp 205-306

Padoa-Schioppa C, Li CS, Bizzi E (2004) Neuronal activity in the supplementary motor area of monkeys adapting to a new dynamic environment. J Neurophysiol 91(1):449-473

Pellizzer G, Sargent P, Georgopoulos AP (1993) Functional hypotheses for the relation between speed and curvature in drawing movements. Ann Soc Neurosci (in press)

Poggio T, Bizzi E (2004) Generalization in vision and motor control. Nature 431:768-774 
Pollick FE, Sapiro G (1997) Constant affine velocity predicts the $1 / 3$ power law of planar motion perception and generation. Vis Res 37:347-353

Polyakov P, Flash T, Abeles M, Ben-Shaul Y, Drori R, Zoltan N (2001) Analysis of motion planning and learning in monkey scribbling movements. In: Proceedings of the 10th biennial conference of the International Graphonomics Society, Nijmegen, The Netherlands, pp 78-83

Richardson MJA, Flash T (2002) Comparing smooth arm movements with the two-thirds power law and the related segmented-control hypothesis. J Neurosci 22:8201-8211

Rohrer B, Fasoli S, Krebs HI, Hughes R, Volpe B, Frontera WR, Stein J, Hogan N (2002) Movement smoothness changes during stroke recovery. J Neurosci 22(18):8297-8304

Sapiro G, Tannenbaum A (1994) On affine plane curve evolution. J Func Anal 119:79-120

Schaal S, Ijspeert A, Billard A (2003) Computational approaches to motor learning by imitation. Phil Trans Royal Soc Lond Series B-Biol Sci 358:537-547

Schwartz AB (1992) Motor cortical activity during drawing vovements: single-unit activity during sinusoid tracing. J Neurophysiol 68(2):528-541

Schwartz AB (1993) Primate motor cortex and free arm motor cortical activity during drawing movements: population response during sinusoid tracing. J Neurophysiol 70:28-36

Schwartz AB (1994) Direct cortical representation of drawing. Science 265:540-542

Schwartz AB, Moran DW (1999) Motor cortical activity during drawing movements: Population representation during leminiscate tracing. 5:2705-2718

Shadmehr R, Mussa-Ivaldi FA (1994) Adaptive representation of dynamics during learning a motor task. J Neurosci 14(5):3208-3224

Sharon A, Brandt E, Basri R (2000) Completion energies and scale. IEEE Trans Pattern Anal Mach Intell 22(10):1117-1131

Soechting JF, Flanders M (1997) Flexibility and repeatability of finger movements during typing: analysis of multiple degrees of freedom. J Comput Neurosci 4:29-46

Soechting JF, Terzuolo CA (1987a) Organization of arm movements in three-dimensional space, wrist motion is piecewise planar. Neuroscience 23(1):53-61
Soechting JF, Terzuolo CA (1987b) Organization of arm movements. motion is segmented. Neuroscience 23:39-51

Sosnik R, Hauptmann B, Karni A, Flash T (2004) When practice leads to co-articulation: the evolution of geometrically defined movement primitives. Exp Brain Res 156:422-438

Spain B (1957) Analytical conics. Pergamon

Spivak M (1979) A comprehensive introduction to differential geometry. Publish or Perish, Berkeley

Stein RB, Oguztoreli MN, Capady C (1985) What is optimized in muscular movements? In: Jones NL, McCartney N, McComas AJ (eds) Multiple muscle systems. Biomechanics and movement. Kinetic Publishers pp 283-301

Sternad D, Schaal S (1999) Segmentation of endpoint trajectories does not imply segmented control. Exp Brain Res 124:118-136

Tanaka M, Iriki A, Iwamura Y (1996) Coding of modified body schema during tool use by macaque postcentral neurons. Neuroreport 7:2325-2330

Todd JT, Oomes AHJ, Koenderink JJ, Kappers AML (2001) On the affine structure of perceptual space. Psychol Sci 12:191-196

Viviani P (1986) Do units of motor action really exist? In: Heuer H, Fromm C (eds) Generation and modulation of action patterns. Springer, Heidelberg, pp 201-216

Viviani P, Cenzato M (1985) Segmentation and coupling in complex movements. J Exp Psychol: Human Percept Perform 11:828-845

Viviani P, Flash T (1995) Minimum jerk, two-thirds power law and isochrony: converging approaches to motion planning. J Exp Psychol: Human Percept Perform 21:32-53

Viviani P, Stucchi N (1989) The effect of movement velocity on form perception:geometric illusions in dynamic displays. Percept Psychophys 46:266-274

Viviani P, Stucchi N (1992) Biological movements look uniform: evidence of motor-perceptual interactions. J Exp Psychol: Human Percept Perform 18:603-623

Von-Hofsten C (1991) The structuring of early reaching movements: a longitudinal study. J Motor Behav 23:280-292

Wolpert DM, Ghahramani Z (2000) Principles of movement neuroscience. Nature Neurosci 3:1212-1217 